\title{
Discovery of a Significant Magnetic Cataclysmic Variable Population in the Limiting Window
}

\section{Citation}

Hong, JaeSub, Maureen van den Berg, Jonathan E. Grindlay, Mathieu Servillat, and Ping Zhao. 2012. "DISCOVERY OF A SIGNIFICANT MAGNETIC CATACLYSMIC VARIABLE POPULATION IN THE LIMITING WINDOW." The Astrophysical Journal 746 (2): 165. https:// doi.org/10.1088/0004-637x/746/2/165.

\section{Permanent link}

http://nrs.harvard.edu/urn-3:HUL.InstRepos:41399783

\section{Terms of Use}

This article was downloaded from Harvard University's DASH repository, and is made available under the terms and conditions applicable to Other Posted Material, as set forth at http:// nrs.harvard.edu/urn-3:HUL.InstRepos:dash.current.terms-of-use\#LAA

\section{Share Your Story}

The Harvard community has made this article openly available. Please share how this access benefits you. Submit a story.

Accessibility 


\title{
DISCOVERY OF A SIGNIFICANT MAGNETIC CATACLYSMIC VARIABLE POPULATION IN THE LIMITING WINDOW
}

\author{
JaeSub Hong $^{1}$, Maureen van den Berg ${ }^{2}$, Jonathan E. Grindlay ${ }^{1}$, Mathieu Servillat $^{1}$, And Ping ZhaO $^{1}$ \\ ${ }^{1}$ Harvard-Smithsonian Center for Astrophysics, 60 Garden Street, Cambridge, MA 02138, USA; jaesub@ head.cfa.harvard.edu \\ ${ }^{2}$ Astronomical Institute, Utrecht University, Princetonplein 5, Utrecht, 3508 TA, The Netherlands \\ Received 2011 March 1; accepted 2011 December 1; published 2012 February 3
}

\begin{abstract}
We have discovered 10 periodic X-ray sources from the 1 Ms Chandra ACIS observation of the Limiting Window $(\mathrm{LW})$, a low-extinction region $\left(A_{V} \sim 3.9\right)$ at 1.4 south of the Galactic center. The observed periods $(\sim 1.3-3.4 \mathrm{hr})$ and the X-ray luminosities $\left(10^{31.8-32.9} \mathrm{erg} \mathrm{s}^{-1}\right.$ at $\left.8 \mathrm{kpc}\right)$ of the 10 periodic sources, combined with the lack of bright optical counterparts and thus high X-ray-to-optical flux ratios, suggest that they are likely accreting binaries, in particular, magnetic cataclysmic variables (MCVs). All of them exhibit a relatively hard X-ray spectrum $(\Gamma<2$ for a power-law model), and relatively high extinction observed in the X-ray spectra of at least five sources indicates some intrinsic absorption in the system, which is also a typical sign of MCVs. On close inspection, the period distribution of these sources resembles those of polars, whereas the relatively hard spectra suggest that they could be intermediate polars (IPs). These puzzling properties can be explained by unusual polars with buried magnetic fields or a rare sub-class of MCVs, nearly synchronous MCVs. These unusual MCVs may provide important clues in the evolutionary path of MCVs from IPs to polars. The completeness simulation indicates that $\gtrsim 40 \%$ of the hard $\mathrm{X}$-ray sources in the LW are periodic. Therefore, this discovery provides a first direct evidence of a large MCV population in the bulge and further supports the current view that MCVs constitute the majority of low-luminosity hard X-ray sources $\left(\sim 10^{30-33} \mathrm{erg} \mathrm{s}^{-1}\right)$ in the bulge.
\end{abstract}

Key words: Galaxy: bulge - novae, cataclysmic variables $-\mathrm{X}$-rays: binaries

Online-only material: color figures

\section{INTRODUCTION}

The high sensitivity and superb spatial resolution of Chandra enabled a population study of low-luminosity X-ray sources $\left(L_{\mathrm{X}} \sim 10^{30-34} \mathrm{erg} \mathrm{s}^{-1}\right)$ on Galactic scales beyond the local solar neighborhood. Several ongoing campaigns, including our own Chandra Multi-wavelength Plane (ChaMPlane) survey (Grindlay et al. 2005), aim to improve the census of the Galactic low-luminosity X-ray sources. The Galactic bulge, in particular, has been of great interest. More than 3000 discrete X-ray sources have been discovered in the $17^{\prime} \times 17^{\prime}$ region around Sgr A* (Muno et al. 2003a, 2009, hereafter M03a, M09). In ChaMPlane, we study the bulge through our dedicated surveys of low-extinction bulge regions ("Windows survey") and a latitudinal strip around the Galactic center ("Bulge Latitude Survey"), where thousands of X-ray sources have also been discovered (J. E. Grindlay et al. 2012, in preparation; Hong et al. 2009a, hereafter H09a). Multi-wavelength follow-up of these $\mathrm{X}$-ray sources has been ongoing for source classification through optical/infrared imaging and spectroscopy (e.g., Koenig et al. 2008; van den Berg et al. 2006, 2009, hereafter B06, B09).

H09a (see also M03a) have found that the X-ray sources in the Galactic center region (GCR) show largely homogeneous X-ray properties (e.g., intrinsically hard X-ray spectra, photon powerlaw index $\Gamma<1$ for a power-law model). The lack of bright-IR $(K<15)$ counterparts for the GCR X-ray sources indicates that high-mass X-ray binaries, once considered a major candidate for bulge X-ray sources, cannot account for more than $10 \%$ of the population (Laycock et al. 2005, hereafter L05). Currently, the leading candidates that fit the observed properties are magnetic cataclysmic variables (MCVs; L05; M09; H09a).

Recently we started a search for periodic ChaMPlane sources (e.g., Hong et al. 2009b, hereafter H09b), in part to circumvent difficulties in source identification, which arise from large distances, high extinction (except for the Windows fields), and source confusion due to high stellar density in the bulge. In this work, we report the discovery of 10 periodic X-ray sources from the 1 Ms Chandra exposure of the Limiting Window (LW), a low-extinction region at 1.4 south of the Galactic center (see also H09a).

The low-extinction Window fields, including the LW, provide a rare opportunity for studying the GCR source population without obscuration from molecular clouds (H09a; H09b; B06; B09). Revnivtsev et al. (2009, hereafter R09) showed that the Galactic ridge X-ray emission (GRXE), the nature of which has been puzzling for decades, is mainly made up of discrete faint sources of known nature, primarily active binaries (ABs) or cataclysmic variables (CVs), based on the Chandra observations of the LW. However, the exact composition of the discrete sources in the GRXE still remains unresolved (see also Revnivtsev et al. 2011). We explore the X-ray and optical properties of the 10 periodic X-ray sources in the LW, their implication for evolutionary models of MCVs, and their connection to thousands of X-ray sources in the GCR.

\section{X-RAY OBSERVATION AND TIMING ANALYSIS}

\subsection{Chandra Observation and Source Search}

The LW was observed for a total of $1 \mathrm{Ms}$ exposure (100 ks in 2005 and $900 \mathrm{ks}$ in 2008) with the Chandra ACIS-I instrument (H09a; R09). Table 1 lists the observational history of the field. The X-ray data were analyzed as a part of our ongoing ChaMPlane survey (Grindlay et al. 2005), and the analysis procedures are described in detail in Hong et al. (2005, hereafter H05; see also H09a). In summary, we stacked all of the 13 separate pointings and searched for discrete sources in the 
Table 1

Chandra ACIS-I Observations of the LW

\begin{tabular}{|c|c|c|c|c|c|c|c|}
\hline ObsID & $\begin{array}{c}\text { Start Time } \\
\text { (UT: yyyy } / \mathrm{mm} / \mathrm{dd} \mathrm{h}: \mathrm{m})\end{array}$ & $\begin{array}{c}\text { R.A. } \\
\left({ }^{\circ}\right)\end{array}$ & $\begin{array}{c}\text { Decl. } \\
\left({ }^{\circ}\right)\end{array}$ & $\begin{array}{c}\text { Roll } \\
\left({ }^{\circ}\right)\end{array}$ & $\begin{array}{c}\mathrm{GTI}^{\mathrm{a}} \\
(\mathrm{ks})\end{array}$ & $\begin{array}{l}\text { Exposure } \\
(\mathrm{ks})\end{array}$ & $\begin{array}{l}\text { Epoch }^{4} \\
\text { (duration) }\end{array}$ \\
\hline 6362 & $2005 / 08 / 1916: 15$ & 267.86875 & -29.58800 & 273 & 37.4 & 38 & \\
\hline \multirow[t]{2}{*}{5934} & 2005/08/22 08:16 & 267.86875 & -29.58800 & 273 & 36.1 & 41 & \\
\hline & & & & Subtotal & 73.5 & 79 & 1 (3.13 days) \\
\hline \multirow[t]{2}{*}{6365} & $2005 / 10 / 2514: 55$ & 267.86875 & -29.58800 & 265 & 20.4 & 21 & \\
\hline & & & & Subtotal & 20.4 & 21 & 2 (0.24 days) \\
\hline 9505 & $2008 / 05 / 07$ 15:29 & 267.86375 & -29.58475 & 82 & 10.7 & 11 & \\
\hline \multirow[t]{2}{*}{9855} & 2008/05/08 05:00 & 267.86375 & -29.58475 & 82 & 55.3 & 57 & \\
\hline & & & & Subtotal & 66.0 & 68 & 3 (1.22 days) \\
\hline 9502 & 2008/07/17 15:45 & 267.86375 & -29.58475 & 281 & 161.7 & 167 & \\
\hline 9500 & $2008 / 07 / 2008: 11$ & 267.86375 & -29.58475 & 280 & 158.6 & 165 & \\
\hline 9501 & $2008 / 07 / 23$ 08:13 & 267.86375 & -29.58475 & 279 & 130.0 & 135 & \\
\hline 9854 & $2008 / 07 / 2705: 53$ & 267.86375 & -29.58475 & 278 & 22.4 & 25 & \\
\hline 9503 & $2008 / 07 / 2817: 37$ & 267.86375 & -29.58475 & 275 & 99.5 & 103 & \\
\hline 9892 & 2008/07/31 08:07 & 267.86375 & -29.58475 & 275 & 64.8 & 65 & \\
\hline 9893 & 2008/08/01 02:44 & 267.86375 & -29.58475 & 275 & 41.4 & 45 & \\
\hline \multirow[t]{2}{*}{9504} & 2008/08/02 21:23 & 267.86375 & -29.58475 & 275 & 122.8 & 127 & \\
\hline & & & & Subtotal & 801.2 & 832 & 4 (17.7 days) \\
\hline
\end{tabular}

Notes.

${ }^{a}$ The selected Good Time Interval (GTI) that is based on the lack of high background fluctuations $(<3 \sigma)$. See Hong et al. (2005).

b The observations are grouped into four separate epochs (durations given in days), so that the total span of each epoch does not exceed a month.

$0.3-2.5,2.5-8.0$, and $0.3-8.0 \mathrm{keV}$ band images, using the wavdetect routine (Freeman et al. 2002). The source lists of the three energy bands were then cross-checked to produce a list of 1397 unique discrete sources based on the positional uncertainty of each source. Carefully designed aperture photometry (H05) that takes into account source crowding was applied to each source in the $0.5-2.0,2.0-8.0$, and $0.5-8.0 \mathrm{keV}$ bands. The complete source list and their photometries will be presented elsewhere (J. Hong et al. 2012, in preparation) along with comparison of the results from two popular source detection algorithms, wavdetect and wvdecomp. ${ }^{3}$ In this paper, we use the sources discovered by the wavdetect routine. Since we can identify periodic X-ray modulation only from relatively bright sources, the two search algorithms make no difference for the results of this paper. The catalog of the 319 sources and their photometry results from the initial $100 \mathrm{ks}$ exposure can be found in H09a.

\subsection{X-Ray Timing Analysis}

The 1 Ms Chandra exposure of the LW can be divided into four epochs so that the total span of each epoch does not exceed a month, which allows a period search in a single ephemeris (Table 1). Of the four epochs, the last epoch provides a long exposure (GTI: $801 \mathrm{ks}$ ) suitable for an in-depth search of periodic modulation. For periodicity search, we selected the 381 sources with background-subtracted net counts greater than 100 in the $0.3-8.0 \mathrm{keV}$ band in Epoch 4 (Figure 1).

Photon arrival times of each source were barycenter corrected to barycentric dynamical time (TDB) by the axbary routine in the CIAO tool (ver 3.4). Then, we generated a backgroundsubtracted light curve of each source in $12.8 \mathrm{~s}$ bins, and we applied a Lomb-Scargle (LS) routine (Scargle 1982) to the light curve to search for periodic X-ray modulation. We subtracted the background counts in the source aperture region using the

3 By A. Vikhlinin; http://hea-www.harvard.edu/RD/zhtools/. See also M09.

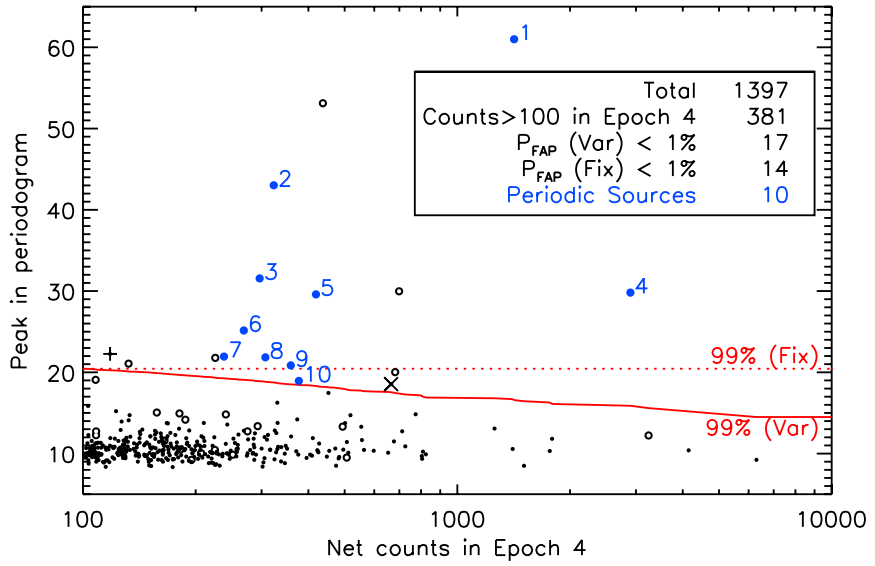

Figure 1. Distribution of the background-subtracted counts in Epoch 4 of the sources and the peak values of the periodograms by the LS method. Of 17 sources with $P_{\text {FAP }}$ (Var) $<1 \%$ (net count-dependent false alarm probability; see Section 2.3 for its definition) or $P_{\text {conf }}$ (Var) $>99 \%$ (red solid), we identify 10 periodic sources (see Section 3). The periodicities of five sources (open circles) are false, originating from either the dithering motion of the instrument or non-periodic X-ray variabilities (e.g., flares). We also exclude two other sources ("+" and " $x$ ") because of the marginal nature of their detections (see Section 3).

(A color version of this figure is available in the online journal.)

events, with a proper scale, that fell in the background annulus region around the source, which excludes the source regions of neighbors (H05). The $12.8 \mathrm{~s}$ bin interval was chosen for efficient executions of the LS routine over a long exposure, and it only suppresses short periods below $\sim 100 \mathrm{~s}$ (see Section 6.1 and Figure 7), where there is already a concern due to the CCD readout time $(\sim 3.2 \mathrm{~s})$. Randomization of photon arrival times to compensate for fixed CCD readout cycle makes practically no difference for the search results owing to the longer time bins used for the light curves and the long exposure consisting of multiple pointings, where multiple phases of $\mathrm{CCD}$ readout 
cycle produce an effect similar to randomizing arrival times to some degree.

The search periods were selected successively, starting from the total duration $(T)$ down to $10^{-4} T$ by a decrement of $\Delta P=P^{2} /\left(2 T s_{f}\right)$, where we introduced an oversampling factor, $s_{f}\left(s_{f}=1\right.$ means no oversampling), in order to sample the periodograms relatively smoothly over the entire period search range. We change $s_{f}$ logarithmically from 1.0 at the shortest period $\left(10^{-4} T\right)$ to 4.0 at the longest $(T)$, and a relative increase in the number of search periods due to the introduction of $s_{f}$ is about $30 \%$. In Epoch 4 where the total duration spans about 17.7 days with a combined total exposure of $832 \mathrm{ks}$, we searched 26,499 periods from about 153 s to 17.7 days. In this analysis, we further limit the search range between $153 \mathrm{~s}$ and $10 \mathrm{hr}$, which contains 19,911 independent periods out of 26,247 trial periods. This range covers most of the spin and orbital periods of MCVs. We found that apparent periodicities of $>10 \mathrm{hr}$ indicated by the periodograms are false alarms, usually caused by short- or long-term X-ray variabilities (e.g., flares). For selected periodic sources (Section 2.3), we also extended the period search range down to $20 \mathrm{~s}$ in an attempt to find any secondary modulation at periods shorter than $153 \mathrm{~s}$, although the bin size $(12.8 \mathrm{~s})$ of the light curves may suppress detection of short periods below $\sim 100$ s (see Figure 7).

For candidate periodic sources, we also applied an Epoch Folding (EF) method (Leahy et al. 1983) to refine the periods and compare the significance of the primary periods with their harmonics. For the EF method, we generated a backgroundsubtracted folded light curve in 15 bins for a given search period and calculated the $\chi^{2}$ value of the folded light curve with respect to the assumed constant count rate of no periodicity. For each source, we applied the EF method at 1000 periods within $2 \sigma$ of the primary period found by the LS routine, and the same around the half, second, and third harmonics of the primary period.

\subsection{Periodic X-Ray Source Selection}

In order to select valid periodic sources, we have estimated a false alarm probability $\left(P_{\mathrm{FAP}}\right)$ or a confidence level $\left(P_{\text {conf }} \equiv\right.$ $\left.1-P_{\mathrm{FAP}}\right)$. For a given power density $(X)$ in the LS routine, $P_{\text {FAP }}=1-\left(1-e^{-X}\right)^{N_{S} N_{P}}$, where $N_{P}$ is the number of independent trial periods $(19,911)$ and $N_{S}$ is the number of the sources. To validate $P_{\text {FAP }}$ estimated from the above trial statistics, we have also performed $\sim 100,000$ simulations by randomizing photon arrival times. These simulations allow

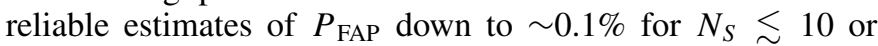
$\sim 1 \%$ for $N_{S} \lesssim 100$, etc., where the simulation results match with those from the trial statistics. For a larger value of $N_{S}$ or a smaller value of $P_{\mathrm{FAP}}$, more simulations are required. In the following, we calculate $P_{\mathrm{FAP}}$ according to the trial statistics.

The search is usually conducted for the brightest sources first, since they have a better chance of periodicity detection. Depending on the search limit of net count $\left(N_{\text {net }}\right)$, which determines $N_{S}, P_{\text {FAP }}$ changes. Although the LS routine is known to be more sensitive to low count sources than a few other conventional Fourier analyses (e.g., see Chapter 6.1 in Bretthorst 1988), its performance eventually drops out as the net count decreases to zero. Extending the search limit by including low count sources with no chance of periodicity detection can arbitrarily increase $P_{\text {FAP }}$, which can make valid periodic modulation appear insignificant. On the other hand, since search performance depends on various factors (e.g., background counts) besides net counts, it is desirable to extend the search to a relatively low net count to be conservative.
Therefore, we set the search limit at a relatively low value $\left(N_{\text {net }}=100\right)$, and we use two types of the $P_{\text {FAP }}$ estimates: $P_{\text {FAP }}$ (Var), where $N_{S}=N_{S}\left(\geqslant N_{\text {net }}\right.$ ), and $P_{\text {FAP }}$ (Fix), where $N_{S}=N_{S}\left(N_{\text {net }} \geqslant 100\right)=381$. The former provides an initial cut for candidate periodic sources, and the latter provides a list of sources with a higher confidence.

Modulation amplitude is also a good metric for significance of periodicity. In order to describe diverse pulse profiles, we use the rms amplitude $\left(A_{\text {rms }}\right)$ given by

$$
\begin{gathered}
A_{\mathrm{rms}}=\left(\frac{Z_{1}^{2}}{N}\right)^{1 / 2} \frac{N}{N-B}, \\
Z_{1}^{2}=\frac{2}{N}\left\{\left[\Sigma_{j} \cos \phi\left(t_{j}\right)\right]^{2}+\left[\Sigma_{j} \sin ^{2} \phi\left(t_{j}\right)\right]^{2}\right\},
\end{gathered}
$$

where $N$ and $B$ are the total and background number of events in the source aperture region, respectively, $t_{j}$ is the arrival time, and $\phi(t)$ is the phase at time $t$ that would be expected for a given period (Muno et al. 2003b, hereafter M03b, and references therein). For easy comparison with the literature, we also define another modulation amplitude as $A_{M}=1-R_{\min } / R_{\max }$, where $R_{\min }\left(R_{\max }\right)$ is the minimum (maximum) count rate of the folded light curve. Finally, for synthetic light-curve simulations, we use sinusoidal variations, where the light curve is described as $1+A_{0} \sin \phi(t)$, where $0<A_{0} \leqslant 1$ and $\phi(t)$ is the phase of photon arrival time $t$. For sinusoidal modulations, $A_{0}=\sqrt{2} A_{\mathrm{rms}}, A_{M}=$ $2 A_{0} /\left(1+A_{0}\right)$, and $A_{\mathrm{rms}}<A_{0} \leqslant A_{M}$. Since both $R_{\min }$ and $R_{\max }$ depend on the bin size of folded light curves, we calculate $A_{M}$ from $A_{\text {rms }}$ using the above relations.

We also use simulations to calculate a detection probability of periodicity $\left(P_{\text {det }}\right)$. For each candidate periodic source, we generate 1000 synthetic light curves to estimate $P_{\text {det }}$ within about $1 \%$ accuracy. Each synthetic light curve is consistent with a sinusoidal variation of the measured period and modulation amplitude of the source, while accounting for the Good Time Intervals (GTIs) and barycentric shifts of the CCD readout time of the real data. Then we perform the LS search algorithm to see how often we detect the same periodicity with $P_{\text {FAP }}$ (Fix) $\leqslant 1 \%$. These simulations enable completeness correction of periodicity detection (see also Section 6.2) and provide another validity measure of the detection in addition to $P_{\text {FAP. }}$.

Finally, we also performed the Bayesian Block (BB) search for long-term variability (Scargle 1998). In order to apply the $\mathrm{BB}$ search to the data set of a long duration (3 yr) with long exposure gaps $(>1 \mathrm{yr}$ ), we eliminate exposure gaps longer than $20 \mathrm{ks}$ and generate a semi-contiguous series of photon arrival times.

The simulations for $P_{\mathrm{FAP}}$ and $P_{\mathrm{det}}$ along with period search require significant computational power. In order to handle the computational burden efficiently at low cost, we have utilized a GPU-based desktop supercomputer equipped with a C1060 Tesla GPU ( 240 cores) from Nvida. ${ }^{4}$ A simple code conversion using an IDL ${ }^{5}$ GPU library $\left(\right.$ gpulib $\left.^{6}\right)$ has boosted the speed of some routines by a factor of 10 in comparison with the regular IDL routines. ${ }^{7}$

\footnotetext{
4 http://www.nvidia.com

5 http://www.ittvis.com/idl

6 http://txcorp.com/products/GPULib

7 The complete code optimization is expected to boost the speed by another factor of 10 according to the examples given by gpulib.
} 

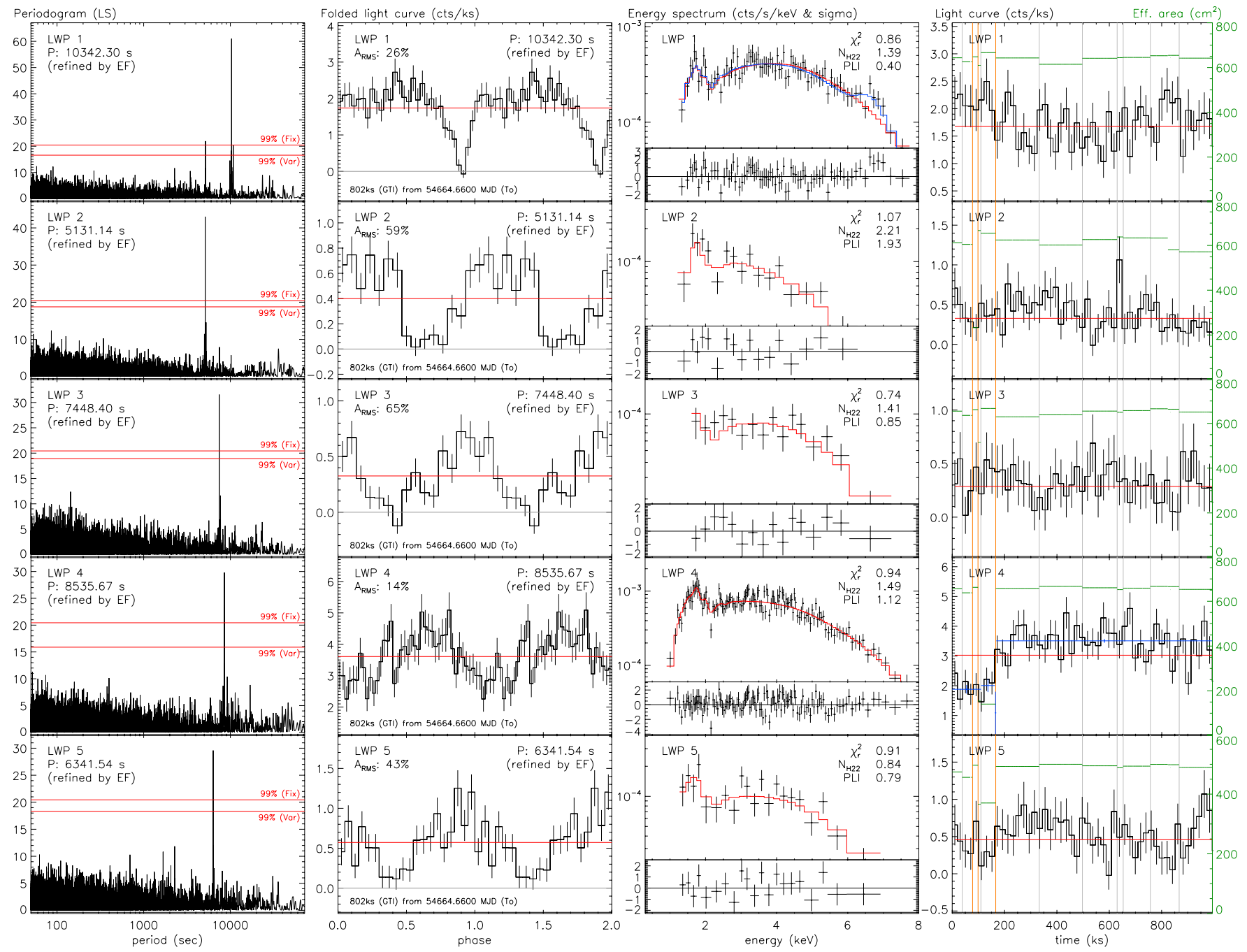

Figure 2. Periodograms, folded light curves, energy spectra, and compressed light curves of the periodic X-ray sources (LWP 1-5) in the LW. The periodograms are based on the LS method, and the 99\% confidence levels for $N_{S}=381$ (Fix) and $N_{S}=N_{S}\left(>N_{\text {net }}\right.$ ) (Var) are shown by (red) horizontal lines (see Section 2.3). The folded light curves are drawn with $T_{0}=$ MJD 54664.6600, and the (red) horizontal lines show the average rates. In the energy spectra, the (red) lines show the results of simple power-law model fits. In the case of LWP 1, a model fit using a power law plus an iron line is shown in blue. In the compressed light curves, the (red) horizontal lines represent the average rate, the (green) steps show the effective area of each pointing, and the vertical lines indicate the epoch boundaries (yellow) and exposure gaps (gray, $>20 \mathrm{ks}$ ). For LWP 4, the Bayesian Blocks are shown by blue lines.

(A color version of this figure is available in the online journal.)

\section{X-RAY ANALYSIS RESULTS}

Figure 1 illustrates the search results and the periodic source count. For initial screening, we select 17 sources that exhibit a periodicity with $P_{\text {FAP }}$ (Var) less than $1 \%$. We exclude five sources with apparent periodicity owing to either the dithering motion of the telescope $(707,1000 \mathrm{~s}$, and their immediate (sub-) harmonics) or non-periodic variabilities (e.g., flares). In addition, we exclude J175055.5-292948 (period: 5331 s) since its $P_{\text {det }}$ is only $0.1 \%$ ("+" in Figure 1). For sources with $P_{\text {FAP }}$ (Fix) $\geqslant 1 \%$, we only consider them periodic if they show another significant indicator of periodicity (e.g., the results of the EF method, see Section 5.10), and so J175103.9-293430 (period: $3821 \mathrm{~s}$ ) is also excluded (" $\times$ " in the figure). The final list of the periodic sources in the LW contains 10 sources (nine with $P_{\text {FAP }}$ (Fix) $<1 \%$ ). The spatial distribution of the 10 periodic sources does not show any obvious sign of clustering or pattern.

Table 2 lists the basic results of period search and photometry of the 10 periodic sources in the LW. For easy reference, in addition to the X-ray source name (starting with CXOPS, see
H05), we assign an abbreviated version of the name starting with prefix "LWP" indicating "Limiting Window Periodic sources." The number IDs in the short name are assigned in the reverse order of the periodogram value at the observed periods (i.e., the likelihood of true periodicity). Note that B09 list the sources with prefix "LW."

Figures 2 and 3 show the periodograms, the folded light curves, the X-ray spectra with model fits, and the compressed light curves without long exposure gaps of the 10 periodic sources. The periodograms are based on the LS method, which was applied to the binned light curves of Epoch 4. The folded light curves are also generated from the data obtained in Epoch 4 and are very similar to the ones from the full $1 \mathrm{Ms}$ exposure (not shown). The periodogram and the folded light curve of LWP 7 are from the last $352 \mathrm{ks}$ GTI in 8.1 days when the source was observed sufficiently distant from any CCD edges.

The X-ray spectrum of each source is generated using all of the available data, and each spectral bin contains more than 20 background-subtracted net counts. The red solid lines show the results using a simple power model fit, and for LWP 1, 
Table 2

Periodic Sources in the LW

\begin{tabular}{|c|c|c|c|c|c|c|c|c|c|}
\hline $\begin{array}{l}1) \\
\text { ID } \\
\text { LWP }\end{array}$ & $\begin{array}{c}\text { (2) } \\
\text { Source Name } \\
\text { CXOPS }\end{array}$ & $\begin{array}{c}(3) \\
\text { Counts (Epoch 4) } \\
(0.3-8 \mathrm{keV})\end{array}$ & $\begin{array}{l}\text { (4) } \\
\text { Period } \\
\text { (s) }\end{array}$ & $\begin{array}{c}(5) \\
P_{\text {FAP }} \text { (Fix) } \\
\left(10^{-n}\right)\end{array}$ & $\begin{array}{c}(6) \\
A_{\mathrm{RMS}} \\
(\%)\end{array}$ & $\begin{array}{l}(7) \\
P_{\text {det }} \\
(\%)\end{array}$ & $\begin{array}{l}(8) \\
\text { Offset } \\
\left({ }^{\prime}\right)\end{array}$ & $\begin{array}{c}(9) \\
\text { Signi. } \\
\text { Harmonics }\end{array}$ & $\begin{array}{l}(10) \\
\text { Notes }\end{array}$ \\
\hline 1 & $\mathrm{~J} 175151.2-293310$ & $1418(41)$ & $10342(5)$ & 19.6 & $26(3)$ & 99.8 & 5.6 & & \\
\hline 2 & J175123.5-293755 & $323(21)$ & $5131(5)$ & 11.8 & $59(5)$ & 99.9 & 2.6 & & \\
\hline 3 & J175129.1-292924 & $296(22)$ & $7448(10)$ & 6.8 & $65(6)$ & 99.1 & 6.1 & & \\
\hline 4 & J175131.6-292956 & $2899(56)$ & $8536(14)$ & 6.1 & $14(2)$ & 83.3 & 5.6 & & BB: 4 \\
\hline 5 & J175133.9-292754 & $419(29)$ & $6342(7)$ & 6.0 & $43(4)$ & 67.5 & 7.7 & & \\
\hline 6 & J175118.7-293811 & $269(19)$ & $4729(2)$ & 4.0 & $50(5)$ & 80.1 & 3.3 & & \\
\hline 7 & J175122.7-293436 & $119(13)^{\dagger}$ & $12076(91)$ & 2.6 & $60(8)$ & 25.7 & 1.5 & 2 & Edge: $352 \mathrm{ks}$ \\
\hline 8 & J175147.4-294215 & $307(28)$ & $4890(6)$ & 2.4 & $40(4)$ & 9.6 & 7.9 & & \\
\hline 9 & J175133.6-293313 & $359(21)$ & $6597(10)$ & 2.1 & $39(5)$ & 66.1 & 2.6 & 3 & PO:0.91 \\
\hline 10 & J175119.4-293659 & $377(22)$ & $5262(2)^{\ddagger}$ & 1.4 & $30(5)$ & 13.9 & 2.4 & 2 & Second harmonic \\
\hline
\end{tabular}

Notes. Columns: (1) An abbreviated X-ray source ID. (2) The Chandra source name. (3) The background-subtracted net counts in the $0.3-8 \mathrm{keV}$ band in Epoch 4 (832 ks exposure). ${ }^{\dagger}$ The counts for LWP 7 are from the last $352 \mathrm{ks}$ GTI, which is free of near-CCD-edge events (see Section 5.7). See Table 3 for the total net counts of the 1 Ms exposure. (4) The modulation periods are refined by the EF routine around the significant periods found by the LS routine. ${ }^{\ddagger}$ For LWP 10 , the second harmonic (5262 s) is considered the real period (see Section 5.10). (5) The false alarm probability for $N_{S}=381$ (see Section 2.3). (6) The rms modulation amplitude based on Equation (1). (7) The detection probability of periodicity based on simulations using 1000 synthetic light curves for each source. (8) The offset from the aim point of ObsID 5934 (R.A.: $17^{\mathrm{h}} 51^{\mathrm{m}} 28^{\mathrm{s}} .50$, decl.: $-29^{\circ} 35^{\prime} 16^{\prime \prime} .80$ ). (9) The most significant harmonic according to the EF method if the primary period is not. In LWP 10, the second harmonic is significantly more prominent than the primary (see Section 5.10), whereas in the other two sources, the differences are marginal. (10) BB: the number of independent Bayesian Blocks in the light curve if not one (see Section 5.4). Edge: the source falls near a CCD edge in some pointings, and GTI free of such pointings is shown. PO: the $95 \%$ point-spread function (PSF) overlaps with neighbors. The number indicates the fractional radius of the non-overlapping region $(\mathrm{H} 05)$.

Table 3

X-Ray Spectral Properties of the Periodic Sources in the LW Based on Simple Power Model Fits

\begin{tabular}{|c|c|c|c|c|c|c|c|c|c|c|}
\hline \multirow{2}{*}{$\begin{array}{l}(1) \\
\text { Source } \\
\text { ID } \\
\text { LWP }\end{array}$} & \multirow{2}{*}{$\begin{array}{c}\text { (2) } \\
\text { Counts (Total) } \\
(0.3-8 \mathrm{keV})\end{array}$} & \multirow{2}{*}{$\begin{array}{c}(3) \\
\\
E_{50} \\
(\mathrm{keV})\end{array}$} & (4) & (5) & (6) & (7) & \multicolumn{3}{|c|}{$\begin{array}{c}(8) \\
\text { Unabsorbed Flux }\end{array}$} & \multirow{2}{*}{$\begin{array}{c}\text { (9) } \\
\text { Luminosity } \\
0.3-8 \mathrm{keV} \\
\left(10^{\mathrm{x}} \mathrm{erg} \mathrm{s}^{-1}\right)\end{array}$} \\
\hline & & & $N_{\mathrm{H} 22}$ & $\Gamma$ & $\begin{array}{l}\text { EW } \\
(\mathrm{keV})\end{array}$ & $\chi_{v}^{2} /$ dof & \multicolumn{3}{|c|}{$\left(10^{-15} \mathrm{erg} \mathrm{cm}^{-2} \mathrm{~s}^{-1}\right)$} & \\
\hline 1 & $1775(45)$ & $3.86(6)$ & $1.4(2)$ & $0.4(1)$ & & $0.86 / 74$ & $6.3(5)$ & $56(2)$ & $63(2)$ & $30.9-32.7$ \\
\hline & & & $1.7(1)$ & $0.63(2)$ & $0.5(1)$ & $0.74 / 72$ & $8.3(7)$ & $55(1)$ & $64(2)$ & $30.9-32.7$ \\
\hline 2 & 393(23) & $2.9(1)$ & $2.2(5)$ & $1.9(3)$ & & $1.07 / 14$ & $9(1)$ & $8.9(6)$ & 21(1) & $30.4-32.2$ \\
\hline 3 & 361(24) & $3.7(2)$ & $1.4(9)$ & $0.8(4)$ & & $0.74 / 13$ & $1.4(4)$ & $10.2(7)$ & $12.0(8)$ & $30.2-32.0$ \\
\hline 4 & $3217(59)$ & $3.36(4)$ & $1.5(1)$ & $1.12(8)$ & & $0.94 / 123$ & 24(1) & $79(2)$ & 107(2) & $31.1-32.9$ \\
\hline 5 & $473(30)$ & $3.4(2)$ & $0.8(5)$ & $0.8(3)$ & & $0.91 / 18$ & $2.5(4)$ & $12.7(9)$ & $16(1)$ & $30.3-32.1$ \\
\hline 6 & $329(21)$ & $3.2(1)$ & $1.3(6)$ & $1.2(4)$ & & $0.35 / 11$ & $2.6(4)$ & $7.0(5)$ & $10.0(6)$ & $30.1-31.9$ \\
\hline 7 & $295(20)$ & $3.8(1)$ & $0.0(3)$ & $0.0(1)$ & & $1.00 / 10$ & $0.32(6)$ & $7.8(6)$ & $7.6(5)$ & $30.0-31.8$ \\
\hline 8 & $361(30)$ & $3.0(2)$ & $0.7(8)$ & $1.1(5)$ & & $0.83 / 13$ & $1.9(4)$ & $8.1(7)$ & 10.3(9) & $30.1-31.9$ \\
\hline 9 & $457(24)$ & $2.9(1)$ & $0.2(3)$ & $0.8(3)$ & & $1.14 / 17$ & $1.3(1)$ & $8.0(5)$ & $9.3(5)$ & $30.0-31.9$ \\
\hline 10 & $415(23)$ & $3.58(9)$ & $1.6(5)$ & $1.1(3)$ & & $0.57 / 15$ & $3.1(4)$ & $9.7(6)$ & 13.1(7) & $30.2-32.0$ \\
\hline
\end{tabular}

Notes. Columns: (1) An abbreviated X-ray source ID. (2) The background-subtracted net counts in the 0.3-8 keV band from the full 1 Ms exposure. (3) The median energy of the photons in the $0.3-8 \mathrm{keV}$ band. (4) and (5) An estimate of the power-law index $(\Gamma)$ and the absorption $\left(N_{\mathrm{H} 22}\right)$ from spectral model fits, assuming a simple power-law model for the $\mathrm{X}$-ray spectrum in the $0.3-8 \mathrm{keV}$ band. (6) The estimated equivalent width of the iron line from a spectral fit of a power-law model with an iron line at $6.7 \mathrm{keV}$. (7) Reduced $\chi^{2}$ and degrees of freedom (dof) of spectral model fits. (8) An estimate of the unabsorbed flux. (9) The luminosity range in the $0.3-8 \mathrm{keV}$ band for source distance at $1-8 \mathrm{kpc}$.

a spectral fit using a power law plus an iron line at $6.7 \mathrm{keV}$ is also shown (blue). In the compressed light curves, the long exposure gaps ( $>20 \mathrm{ks}$ ) are marked by the vertical lines, and the yellow lines indicate the boundaries of four epochs. The red horizontal lines show the average count rate, and the green lines show the effective area, which varies from pointing to pointing. The blue solid lines for LWP 4 show the count rates of the BBs.

Table 3 summarizes the X-ray spectral properties including estimates of the unabsorbed X-ray fluxes, based on spectral model fits. Note that the net counts in Table 3 are from the full $1 \mathrm{Ms}$ exposure, whereas the net counts in Table 2 are from Epoch 4 except for LWP 7, which shows the net count from the last 352 ks GTI. We fit the spectra of all the sources using a simple power-law model, and for LWP 1, we also tried a power law plus an iron line (see Section 5.1).

\section{OPTICAL OBSERVATION AND PROPERTIES}

We observed the LW field with the Hubble Space Telescope (HST) and Magellan telescope. Five of the 10 sources were in the HST/Advanced Camera for Surveys (ACS) fields (B09), and three of them were reported as possible candidates for accreting binaries or CVs. Table 4 summarizes the combined optical and $\mathrm{X}$-ray properties of the candidate optical counterparts of the Chandra sources. For easy reference, the short names used by 

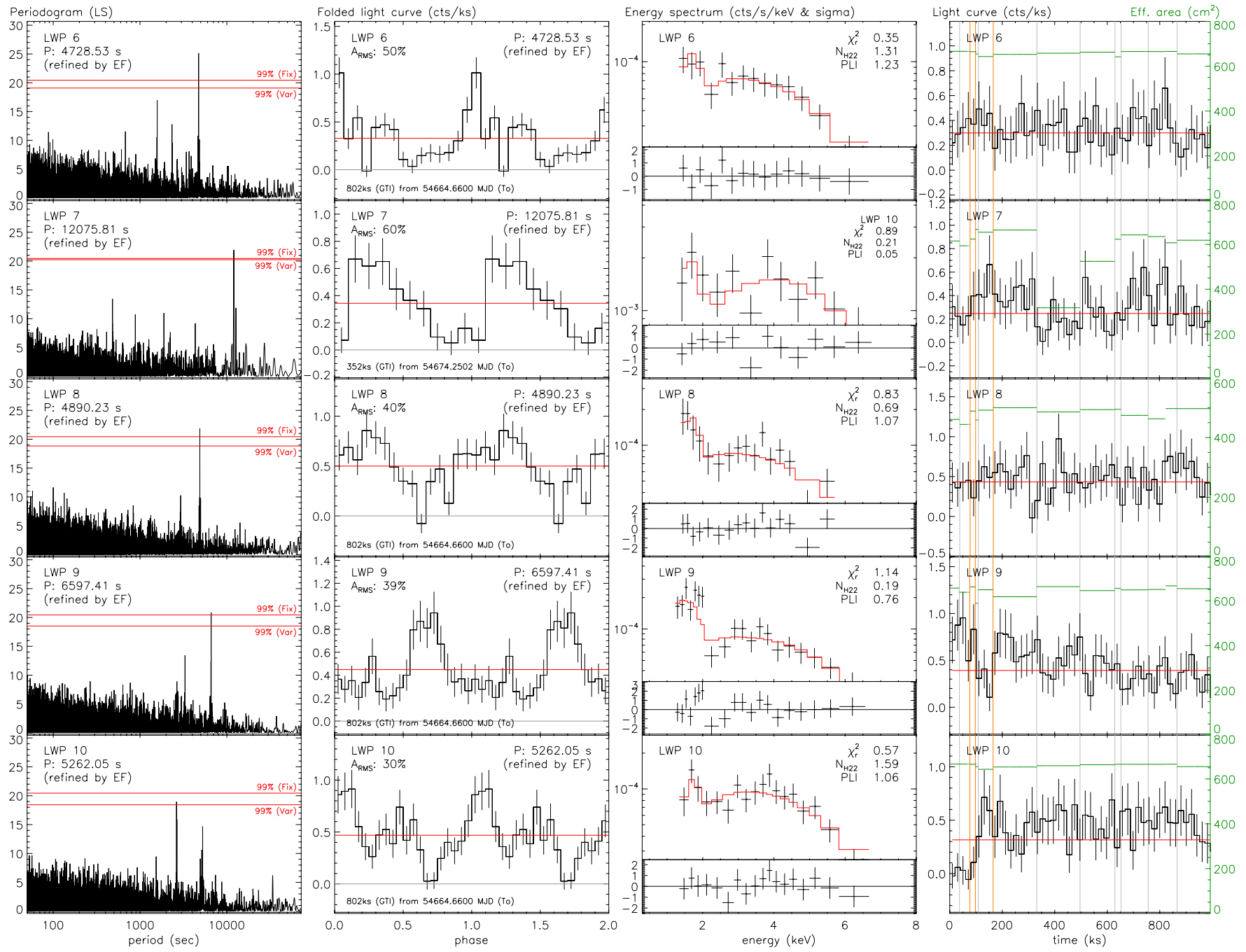

Figure 3. Same as Figure 2 for LWP 6-10. The folded light curve of LWP 7 is drawn with $T_{0}=$ MJD 54674.2502.

(A color version of this figure is available in the online journal.)

Table 4

Candidate Optical Counterparts of the Periodic Sources in the LW

\begin{tabular}{|c|c|c|c|c|c|c|c|c|c|}
\hline $\begin{array}{l}\text { (1) } \\
\text { Source }\end{array}$ & $\begin{array}{l}(2) \\
R\end{array}$ & $\begin{array}{c}(3) \\
B-R\end{array}$ & $F_{\mathrm{X}}(C$ & $\begin{array}{l}\text { 4) } \\
-8 \mathrm{keV})\end{array}$ & $\log$ & $\begin{array}{l}\text { 5) } \\
\left.\mathrm{x} / F_{R}\right)\end{array}$ & No. of & ndidates & $\begin{array}{c}(7) \\
A_{\mathrm{F} 625 \mathrm{~W}}\end{array}$ \\
\hline LWP & (mag) & & (observed) & (unabsorbed) & (observed) & (unabsorbed) & $H S T$ & Magellan & (offset) \\
\hline 1 & $>22.3$ & & $51(1)$ & $64(2)$ & $>1.4$ & $>-0.1$ & & & $3.9\left(1^{\prime} .5\right)$ \\
\hline 2 & $22.3-24.4$ & & $8.0(5)$ & $21(1)$ & $0.6-1.4$ & -0.4 to 0.4 & 2 (LW25) & & $3.6\left(0^{\prime} .1\right)$ \\
\hline 3 & $>22.2$ & & $9.2(6)$ & $12.0(8)$ & $>0.6$ & $>-1.0$ & & & $4.2\left(1^{\prime} .9\right)$ \\
\hline 4 & 23.1(4) & & $75(1)$ & $107(2)$ & $1.9(2)$ & $0.4(2)$ & & 1 & $4.1(1.4)$ \\
\hline 5 & $20.5(2)^{*}$ & & $13.0(8)$ & $16(1)$ & $0.08(8)^{*}$ & $-1.47(8)^{*}$ & & 1 & $4.1(3 ! 4)$ \\
\hline 6 & $22.7(1)$ & 2.9 & $6.8(4)$ & $10.0(6)$ & $0.68(5)$ & $-0.72(5)$ & 5 (LW8) & 1 & $3.9\left(0^{\prime} 3\right)$ \\
\hline 7 & 18.8 & & $7.6(5)$ & $7.6(5)$ & $-0.83(3)$ & $-2.47(3)$ & 1 & 1 & $4.1(0.5)$ \\
\hline 8 & $21.4(2)$ & & $8.1(7)$ & $10.3(9)$ & $0.23(9)$ & $-1.02(9)$ & & 2 & $3.4(3.5)$ \\
\hline 9 & $22.4-25.7$ & & $8.6(5)$ & $9.3(5)$ & $0.7-2.0$ & -0.7 to 0.6 & 6 (LW19) & 1 & $3.6\left(0^{\prime} .2\right)$ \\
\hline 10 & $22.6-24.6$ & & $9.3(5)$ & $13.1(7)$ & $0.8-1.6$ & -0.6 to 0.2 & 4 & & $3.8\left(0^{\prime} \cdot 1\right)$ \\
\hline
\end{tabular}

Notes. Columns: (1) An abbreviated X-ray source ID. (2) $R$ magnitude, based on the HST observation if available; otherwise, based on the Magellan images. For sources with multiple counterparts, the range of those counterparts is given. For sources with no valid counterparts, a lower limit is given, based on the minimum magnitude of neighboring optical sources within a $30^{\prime \prime}$ radius from the X-ray source position. "*”" uses the $I$ magnitude instead of $R$. (3) $B(\mathrm{~F} 435 \mathrm{~W})-R(\mathrm{~F} 625 \mathrm{~W})$ from the $H S T$ observation. (4) The X-ray flux in $10^{-15} \mathrm{erg} \mathrm{cm}^{-2} \mathrm{~s}^{-1}$, from Column 7 in Table 3. (5) $\log \left(F_{\mathrm{X}} / F_{R}\right)=\log \left(F_{\mathrm{X}}\right)+R / 2.5+5.76$. For unabsorbed flux ratios, the $R$ magnitudes are de-reddened by $A_{\mathrm{F} 625 \mathrm{~W}}$. (6) The number of candidate counterparts found in the ACS images of the HST and the Magellan MOSAIC images. Every source in the ACS field of view has more than one counterpart, and for sources reported by B09 their source IDs are given. (7) $A_{\mathrm{F} 625 \mathrm{~W}}$ from Revnivtsev et al. (2010) and the offset of the source from where $A_{\mathrm{F} 625 \mathrm{~W}}$ is sampled. 
B09 are noted for the three sources in Table 4 (e.g., LWP $2=$ LW 25).

\subsection{HST/ACS Data and Analysis}

We observed with HST the inner area of the ACIS field with a $2 \times 2$ mosaic of the Wide-Field Camera (WFC) on the ACS on 2005 August 19. A single WFC pointing images a 3!.4 × 3!.4 field with $\sim 0$ '.05 pixels using two CCD detectors separated by a $2^{\prime \prime} .5$ gap. Exposures were taken through the F435W ( $\left.B_{435}\right)$, F625W $\left(R_{625}\right.$, similar to Sloan $r$ ), and F658N (H $\left.\alpha\right)$ filters. Each tile of the mosaic was observed with the same exposure sequence $4 \times 492 \mathrm{~s}$ in F435W, $168 \mathrm{~s}+2 \times 167 \mathrm{~s}$ in F625W, and $4 \times 496 \mathrm{~s}+4 \times 492 \mathrm{~s}$ in F658N. No dithering was applied to fill in the WFC chip gap. Photometry is performed using a stellar photometry package, DOLPHOT, a modified version of the HSTphot package to do photometry on HST/WFPC2 images (Dolphin 2000). See B09 for the details.

\subsection{Magellan/IMACS Data and Analysis}

On 2007 May 8, we observed the LW field and two other Window fields (Stanek's and Baade's) with the Inamori Magellan Areal Camera and Spectrograph (IMACS) on the $6.5 \mathrm{~m} \mathrm{Mag-}$ ellan (Baade) telescope at Las Campanas, Chile. With seeing 0.8-1".2 FWHM, we obtained a dithered set of five pointings in the $f / 4$ configuration (15.4 field, $0^{\prime \prime} .11$ pixel $^{-1}$ ) to cover an $18^{\prime} \times 18^{\prime}$ region of the $\mathrm{LW}$. This provided a total exposure time of $600,300,180$, and $180 \mathrm{~s}$ in Bessell- $B, V, R$, and CTIO- $I$ filters over the Chandra field, respectively.

We processed the images using standard IRAF tasks and calibrated the astrometry using the Two Micron All Sky Survey catalog as a reference. The astrometric residuals on each CCD frame were $\sim 0^{\prime} .2$. We reprojected and stacked the images using the $\mathrm{SWARP}^{8}$ utility. All frames were normalized to ADU/second units and combined using weight maps constructed from flat fields and bad pixel masks. The initial source search and photometry were performed on the stacked images using DAOPHOT. See also H09b.

\subsection{Optical Matches}

Both the HST and IMACS source lists are boresighted to the Chandra sources as described in Zhao et al. (2005): the boresight correction is less than 0 "' 1 in both R.A. and declination. In search of optical counterparts and their optical properties, for the five sources in the $H S T /$ ACS fields, we use the HST observations. For the other five, we use the Magellan/IMACS observations, where three sources show one or two candidate counterparts. Considering the high stellar density in the region, there is no guarantee of these Magellan/IMACS sources being the true counterparts (e.g., LWP 9 has six candidate counterparts seen in the HST/ACS field, but only one in the Magellan/IMACS image).

The X-ray-to-optical flux ratios, $\log \left(F_{\mathrm{X}} / F_{R}\right)$, in Table 4 are calculated for the $0.3-8 \mathrm{keV}$ band versus the $R$ magnitude. Both the observed and unabsorbed flux ratios are given. For unabsorbed flux ratios, the $R$ magnitude is de-reddened based on the $A_{\mathrm{F} 625 \mathrm{~W}}$ map given by Revnivtsev et al. (2010). For sources with multiple candidate counterparts, a range of the $R$ magnitude and the flux ratio is given, covering all the candidate counterparts. In the case of LWP 2 (= LW 25) and LWP 6 (= LW 8), shown is the $R$ magnitude of the most likely candidate

\footnotetext{
8 http://www.astromatic.net/software/swarp
}

with an unusually blue color (B09). In the case of LWP 8, only one of the candidate counterparts has a measurable $R$ magnitude. For sources with no detectable candidate counterparts, a lower limit for the $R$ magnitude is given, based on the minimum value of optical neighbors within a $30^{\prime \prime}$ radius of the X-ray source position.

The logarithmic flux ratios or their limits of these periodic sources are relatively high: $>-1$ for seven sources and $>-2.5$ for the rest. The intrinsic X-ray-to-optical flux ratios of accreting binaries or active galactic nuclei are usually significantly higher than those of coronal sources (see Section 4.1.2 in B09, and references therein). CVs have the intrinsic flux ratio between -2.5 and +0.5 in the above energy bands, although a few outliers of $\mathrm{ABs}$ or dMe stars have the flux ratios as high as -1 or -0.5 . Therefore, the flux ratio results in Table 4 are consistent with those of CVs.

Note that, except for a few cases with an outstanding blue counterpart, most of the flux ratio values in Table 4 are in fact likely lower limits, considering the high stellar density in the region and the possibility of the true counterpart being fainter and undetected. LWP 5 and 8 have additional uncertainties in their estimates due to the variation in the interstellar absorption across the region: for these sources, the $A_{\mathrm{F} 625 \mathrm{~W}}$ values were sampled about $3^{\prime}$ or $4^{\prime}$ away from the sources. For LWP 7 and 9, the absorption in the X-ray spectra is estimated to be less than what is expected in the field, based on $A_{\mathrm{F} 625 \mathrm{~W}}$. For LWP 3, 5, and 8 , two estimates are consistent, and for the rest, the former is larger than the latter.

\section{SOURCE PROPERTIES}

In this section, we discuss some of the unique properties or analysis caveats of each source.

\subsection{LWP 1: CXOPS J175151.2-293310}

The periodogram reveals significant periodic modulation at the primary period and its half. The folded light curve shows a clear eclipse, but owing to the lack of other features in the folded light curve other than the eclipse, the system can be either a polar or an intermediate polar (IP). The long period suggests that the modulation is likely due to the orbital motion (e.g., only two IPs with $\gtrsim 10^{4} \mathrm{~s}$ spin period in Figure 6(a), see Section 6.1 for the details). If so, the mass of the companion is estimated to be $\sim 0.42 M_{\odot}$ according to Equation (2.89) in Warner (1995). In addition, from the eclipse duration $\Delta \phi \sim 0.09$, the mass ratio $q \gtrsim 0.29$ and the inclination angle $i$ is somewhere between $60^{\circ}$ and $74^{\circ}$ for white dwarf (WD) mass $M_{1} \sim 0.1-1.4 M_{\odot}$ (Equations (2.92) and (2.93) in Warner 1995).

The X-ray spectrum exhibits a feature consistent with an iron line at $6.7 \mathrm{keV}$ (and perhaps $6.4 \mathrm{keV}$ as well). The spectral fit with a power law plus a $6.7 \mathrm{keV}$ line reduces the $\chi_{v}^{2}$ by $14 \%$, compared with a simple power-law model (Table 3 ). In order to estimate the significance of the line (see Protassov 2002), we generated 1000 synthetic spectra, each of which is consistent with a simple power-law model $\left(\Gamma=0.4\right.$ and $\left.N_{\mathrm{H} 22}=1.4\right)$. We then fit each spectrum with a power law plus the iron line and count how many cases reduce the $\chi_{v}^{2}$ by more than $14 \%$. The results were none, indicating that the significance of the line is $99.9 \%$ or higher. The hard X-ray spectrum with an iron emission line suggests that the source is likely an IP (see more about the source type in Section 6.1). 

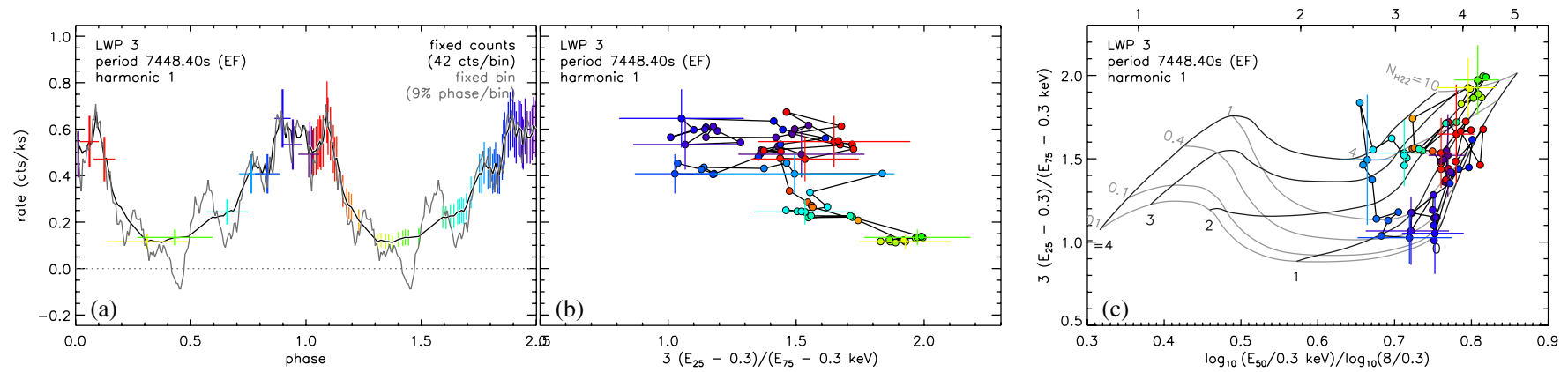

Figure 4. Phase-resolved quantile analysis of LWP 3 using the last 801 ks exposure: (a) the folded light curves, (b) the rate vs. the quartile ratios, and (c) the phase-resolved quantile diagram. The folded light curves are generated using sliding windows of fixed-width phase bins (gray) and variable-width phase bins with fixed net counts (black; example data points with bin sizes are shown in phase $0-1$ ), and the error bars of the latter are color-coded by phase for easy comparison. The quartile ratios are mostly proportional to $\log \left(N_{\mathrm{H} 22}\right)$ for LWP 3 .

(A color version of this figure is available in the online journal.)

\subsection{LWP 2: CXOPS J175123.5-293755}

This source was reported as a possible accreting binary (LW 25) by B09. Two optical sources in the HST/ACS images are found within the error circle of the X-ray source position, and neither of them stands out with any unusual colors. The folded light curve shows a possibility of a long eclipse starting at phase $\sim 0.5$. LWP 2 and 6 are located in the region with an excess of seemingly diffuse soft X-ray background, which perhaps indicates the lowest extinction region of the field. In the case of LWP 2, it shows a significantly larger absorption in the $\mathrm{X}$-ray spectrum than the field average, indicating an intrinsic absorption in the system.

\subsection{LWP 3: CXOPS J175129.1-292924}

This source exhibits a mild anti-correlation between the count rate and the absorption in the X-ray spectrum according to a phase-resolved quantile diagram (Figure 4) (Hong et al. 2004; $\mathrm{H} 09 \mathrm{~b})$. This anti-correlation is consistent with a picture that the observed X-ray modulation is caused by the variation of intrinsic absorption in the system, similar to the IP discovered in BW (H09b). Alternatively, if the X-ray modulation originates from an eclipse or an obscuration of the hot spot or the emission region due to the spin or orbital motion, then the absorption variation may not be expected to be strongly correlated with the rate change. The folded light curve of the source shows a hint of an eclipse (phase $\sim 0.45$ ), which, if true, implies that the system is synchronized (i.e., polar), considering the synchronized primary modulation in the folded light curve.

\subsection{LWP 4: CXOPS J175131.6-292956}

This is the brightest source of the 10 periodic sources. The $\mathrm{X}$-ray spectrum does not show any sign of iron lines. The BB search indicated four independent blocks in the long-term light curve, but since the source fell near a CCD edge in Epoch 3, only two blocks can be credited to be independent. In summary, the observed X-ray flux in 2008 is higher by a factor of $\sim 1.8$ than that in 2005.

\subsection{LWP 5: CXOPS J175133.9-292754}

This source and LWP 8 were observed at relatively large off-axis angles.

\subsection{LWP 6: CXOPS J175118.7-293811}

This source was reported as a candidate CV (LW 8) by B09, based on the high X-ray-to-optical flux ratio and $\mathrm{H} \alpha$ excess (see Figure 4 in B09). The folded light curve in Figure 2 shows a hint of an eclipse at phase $\sim 0.2$. Our routine analysis of phase-resolved quantiles at the primary period and the second harmonic revealed an intriguing result, as illustrated in Figure 5. At each period, we show two versions of folded light curves using sliding phase windows: one with fixed-width phase bins $(10 \%$, gray) and the other with variable-width phase bins but fixed net counts in each phase bin (45 counts, black). The former is better suited to identify a sudden drop in the count rate such as an eclipse, and the latter is better suited to reveal a sudden increase. In addition, the latter is better suited for phase-resolved spectral quantile analysis since every phase bin contains enough events to allow a reliable estimate of energy quantiles. The folded light curves in fixed-width phase bins show an eclipse-like feature both at the primary period (phase $\sim 0.2$ and 0.6 ) and at the second harmonic $(\sim 0.25)$. The ingress and egress of the eclipse are sharper at the second harmonic than the primary period. A narrow eclipse, synchronized with the primary modulation, suggests that the system is a polar.

What is interesting in this source is a spectral change correlated with the phase. At the primary period, the first quarter of the phase shows an intrinsically harder X-ray spectrum $(\Gamma<1)$ than the rest $(\Gamma>1)$, which implies that two different emission regions or mechanisms are present (Figure 5(e)). On the other hand, at the second harmonic, there appears to be a dramatic change in the absorption between the first $\left(N_{\mathrm{H} 22} \gtrsim 2\right)$ and second halves $\left(N_{\mathrm{H} 22} \lesssim 1\right)$ of the phase (Figures $5(\mathrm{~d})$ and (f)). The change in the absorption between two peaks of the folded light curve would suggest that two magnetic poles of the system are visible in turn, and the X-ray emission from one of the two goes through more material (likely an accretion curtain, trailing to a pole and extended from about half of the accretion disk or ring) before reaching us. Figure 5 in Evans \& Hellier (2007) illustrates a possible viewing geometry for such a system: unlike their examples, the soft blackbody component $(\ll 1 \mathrm{keV})$ of LWP 6 is likely always invisible owing to the interstellar absorption, but the geometry allowing for a phase-dependent variation of intrinsic absorption due to an accretion stream or a curtain can apply to LWP 6. This picture, if true, suggests that the second harmonic (9457 s) is a real period and the observed primary period ( $4729 \mathrm{~s})$ is a sub-harmonic, even though the LS method did not find the second harmonic significant. Further observation is required to determine which period represents the true orbital and spin geometry. In addition, a new diagnosis may be required to quantitatively evaluate the significance of 

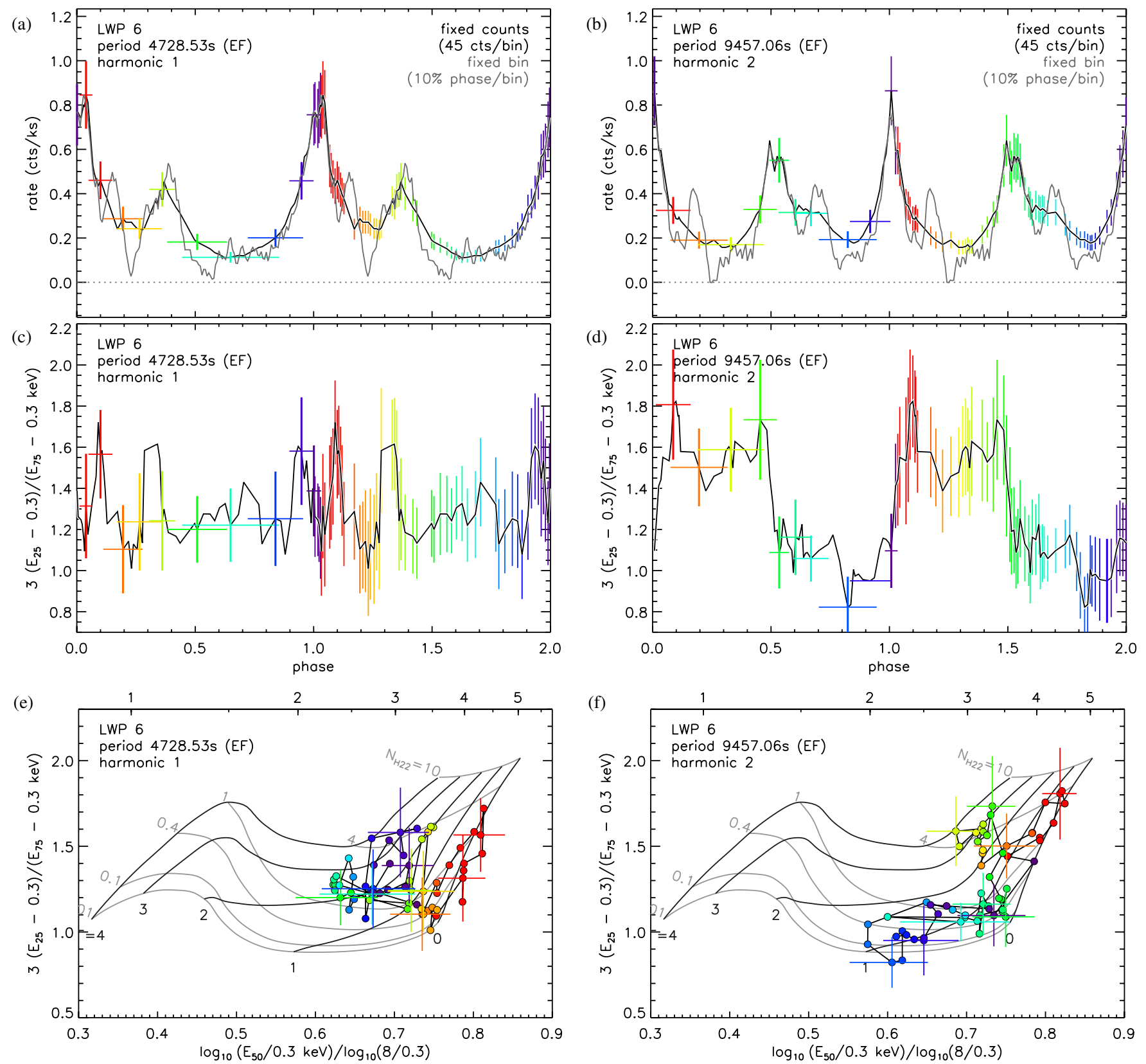

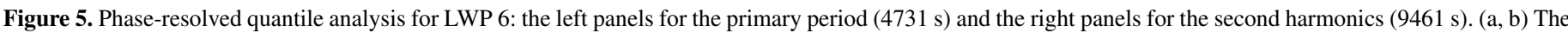

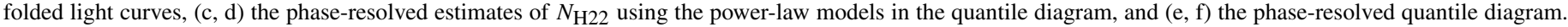
For the $9461 \mathrm{~s}$ period $(\mathrm{d})$, the first half of the phase $(0.0-0.5)$ shows a higher absorption $\left(N_{\mathrm{H} 22} \gtrsim 2\right)$ than the second half $(0.5-1.0)\left(N_{\mathrm{H} 22} \lesssim 1\right)$.

(A color version of this figure is available in the online journal.)

various types of spectral changes (see A. Connors 2012, in preparation).

\subsection{LWP 7: CXOPS J175122.7-293436}

During three pointings out of eight total in Epoch 4, this source fell near a CCD edge (see the effective area in Figure 3), which would discredit the observed periodicity, but the relatively clean data set (the last $352 \mathrm{ks} \mathrm{GTI}$ ) free of near-CCD-edge events also exhibits a significant periodicity at $12,076 \mathrm{~s}$. The net count of LWP 7 in the last $352 \mathrm{ks}$ GTI is only 119 , which is similar to that of J175055.5-292948 ("+" in Figure 1) in Epoch 4, but $P_{\text {det }}$ of LWP 7 is estimated substantially higher than J175055.5292948 because of the relatively lower background counts in the source aperture region of LWP 7. Therefore, we consider the observed periodicity of LWP 7 valid.

\subsection{LWP 8: CXOPS J175147.4-294215}

The folded light curve of the source shows an eclipse-like feature at phase $\sim 0.6$.

\subsection{LWP 9: CXOPS J175133.6-293313}

LWP 9 was reported as a potential accreting binary (LW 19) by B09. The aperture source region (95\% PSF) of these sources mildly overlaps with that of a neighbor. A clean data set relatively free of contamination from the neighbor (see H05 for aperture choice) exhibits the same periodic modulation, so that its periodicity is considered valid. 

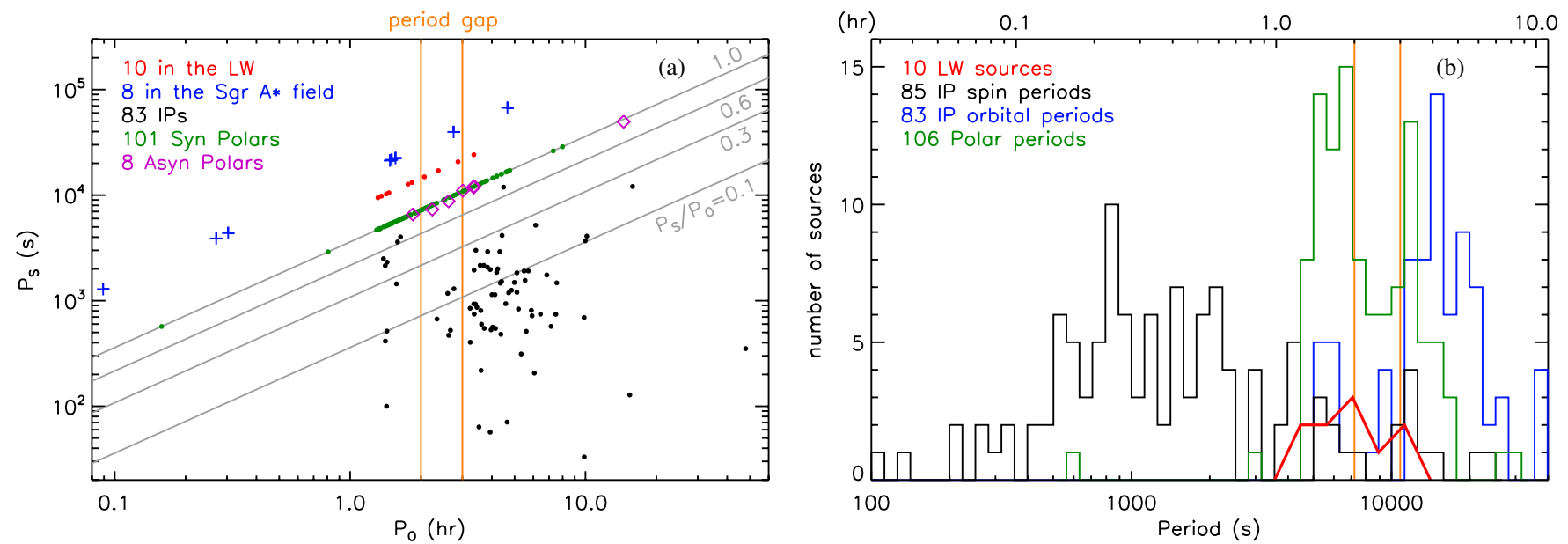

Figure 6. (a) The spin $\left(P_{s}\right)$ vs. orbital period $\left(P_{o}\right)$ distribution of MCVs from the RK catalog (ver 7.15). The vertical yellow lines mark the period gap, and the diagonal lines represent $P_{s} / P_{o}=1,0.6,0.3$, and 0.1 . The purple diamonds show eight asynchronous polars (APs) (three are on top of each other), and there are six near-synchronous IPs with $P_{s} / P_{o}>0.3$. The observed periods of the periodic sources in the LW (red) and the Sgr A* field (blue) are marked as orbital periods, along $P_{s}=2 P_{o}$ or $4 P_{o}$ for clarity (those less than an hour are likely the spin periods). (b) The period distribution of the LW sources (red) is compared with the distribution of the spin (black) and orbital (blue) periods of IPs and the periods (green) of polars in the RK catalog. The periodic sources (red) in the LW fill in the period gap, indicating that these are likely MCVs. The periods of the LW sources are distributed more closely to those of polars (green) than the spin (black) or orbital (blue) periods of IPs.

(A color version of this figure is available in the online journal.)

\subsection{LWP 10: CXOPS J175119.4-293659}

According to the EF analysis for LWP 7, 9, and 10, a higher harmonic exhibits more significant periodicity than the primary period found by the LS method. While the differences in the significance between the primary periods and their harmonics are marginal for two sources, in the case of LWP 10 the second harmonic appears substantially more significant. The $\chi^{2}$ of the 15-bin folded light curve with respect to the constant rate is 133 at the second harmonic versus 89.5 at the primary period. ${ }^{9}$ Therefore, we consider the second harmonic to be the real modulation period for LWP 10 . The high $\chi^{2}$ value at the second harmonic is partially due to an eclipse-like feature in the folded light curve at phase $\sim 0.7$, which suggests that the system is a polar.

\section{DISCUSSION}

In this section, we investigate the most probable source types for the periodic sources found in the LW and their implications, based on the statistical distribution of their properties. We also explore the hidden population of periodic sources in the bulge $\mathrm{X}$-ray sources in the GCR through completeness simulations for periodicity detection.

\subsection{Unusual MCVs?}

The observed source properties, such as the $\mathrm{X}$-ray luminosity range $\left(\sim 10^{30-33} \mathrm{erg} \mathrm{s}^{-1}\right.$ for distance of $\left.1-8 \mathrm{kpc}\right)$, the relatively hard X-ray spectra $(\Gamma<2)$, the period range (1.3-3.4 hr), and the relatively high X-ray-to-optical flux ratios, all indicate that these sources are typical MCVs. But the collection of these properties does not appear to fit well with most common types of MCVs as explained below.

MCVs can be largely divided into two groups, IPs and polars, depending on the relative strength of the magnetic fields.

\footnotetext{
9 For comparison, the expected $\chi^{2}$ of the 15-bin folded light curve is 70.9 at $P_{\text {FAP }}=1 \%$ for $N_{S}=381$ and $N_{P}=19,911$, although the EF search was conducted only for the 10 sources at 4000 periods around the likely true modulation periods.
}

Traditionally, polars are synchronized or nearly synchronous $\left(P_{s} / P_{o} \sim 0.98-1.02\right)$, whereas IPs are not $\left(P_{s} / P_{o} \lesssim 0.1\right)$. Figure 6 shows the spin versus orbital period distribution of the MCVs in the Ritter \& Kolb (RK) catalog (ver. 7.15; Ritter \& Kolb 2003). In Figure 6(a), the observed periods of the periodic $\mathrm{X}$-ray sources found in the $\mathrm{LW}$ and $\mathrm{Sgr} \mathrm{A}^{*}$ fields are shown as orbital periods along $P_{s} / P_{o}=2$ or 4 for easy comparison.

In the case of the periodic sources in the LW, the observed period distribution (red in Figure 6(b)) resembles those of polars (green) better than either the spin (black) or orbital (blue) periods of IPs. Some IPs do have spin or orbital periods at around an hour to three hours, but the majority of the spin (or orbital) periods are shorter (or longer), whereas the majority of the periods of polars are in the same range as those of the periodic sources in the LW.

In order to find out if the above result is due to a perioddependent selection bias in the periodicity search routines, we have conducted a set of simulations using synthetic light curves with various net counts (100-1000), modulation amplitudes $\left(A_{0}=10 \%-100 \%\right)$, and periods $\left(\sim 20-1.1 \times 10^{5} \mathrm{~s}\right)$. For each combination of parameters, we generate 500 synthetic light curves, which allows $\lesssim 2 \%$ accuracy in measurement of the detection probability. As in the simulations in Section 2.3 (and Section 6.2), each synthetic light curve is generated to properly reflect the GTIs and barycentric shifts of the CCD readout time of the real data.

The simulation results show there is no significant selection bias in the range of $150 \mathrm{~s}$ to $10 \mathrm{hr}$ (Figure 7). This implies that the resemblance to the polar period distribution is not due to any selection effect in the search algorithms, ${ }^{10}$ or if these sources are IPs, they indeed belong to a statistically different population from the typical IPs in the RK catalog. In addition to the observed eclipses (or eclipse-like features) synchronized with the primary modulation of the pulsed profiles (e.g., LWP 3, 6, 8, or 10), missing secondary periods from all the sources in Table 2 indirectly support the systems being

\footnotetext{
10 There is, however, a selection bias toward sources with high modulation amplitudes, as expected. See Section 6.2.
} 


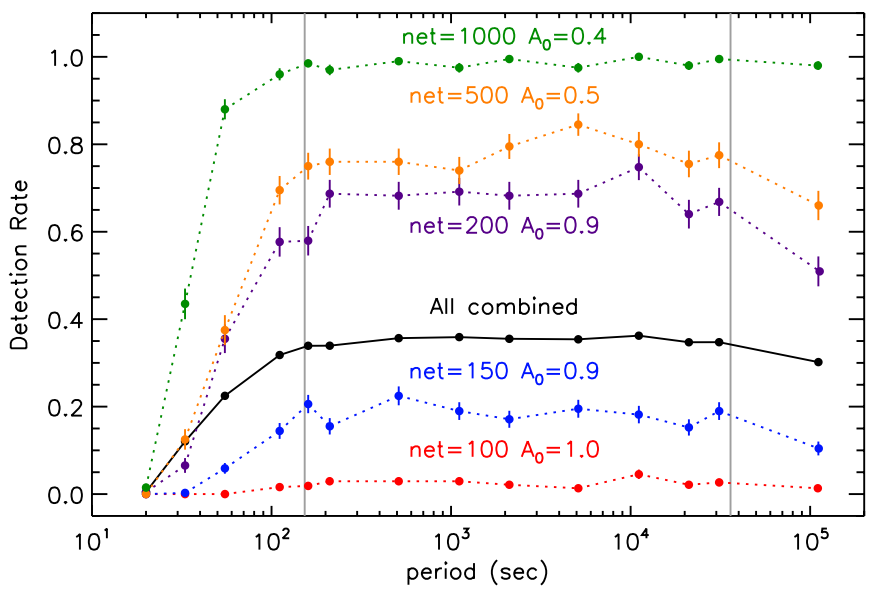

Figure 7. Examples of detection $\left(P_{\mathrm{FAP}}(\mathrm{Fix})<1 \%\right)$ probabilities of periodicity as a function of period by simulations. The plot for "All Combined" includes the following cases: for net $=1000, A_{0}=0.2-0.5$; for net $=500, A_{0}=$ $0.3-0.7$; for net $=200, A_{0}=0.5-1.0$; for net $=150, A_{0}=0.6-1.0$; and for net $=100, A_{0}=1.0$. The detection probability shows no bias within the period range of interest between $150 \mathrm{~s}$ and $10 \mathrm{hr}$ (two vertical gray lines).

(A color version of this figure is available in the online journal.)

polars - synchronized systems, although non-detection does not guarantee the absence of the secondary periods.

Figure 8 illustrates a wide range of modulation amplitudes of the 10 periodic sources in the LW. For comparison, we also show some of the literature-selected IPs and polars. If the X-ray emission originates from a small spot (e.g., polar cap) on the WD surface, the modulation due to the spinning motion of the compact object is expected to exhibit a larger amplitude change than that from the orbital motion. For instance, a sample of IPs in Figure 8, selected from the literature, shows a slightly higher average value of the modulation amplitude at the spin periods (black closed circles) than at the orbital periods (black open circles). However, the modulation distributions of these IPs and polars in Figure 8 are likely selection biased, e.g., 17 polars in Figure 8 are mostly eclipsing systems, which are likely preferentially found in periodicity searches owing to the large modulation amplitude. Note that for polars in Figure $8, A_{M}$ is simply based on $R_{\min }$ and $R_{\max }$ of the published light curves, where by definition $A_{M}=1$ for eclipsing systems, whereas for the periodic sources in the $\mathrm{LW}$, we calculate $A_{M}$ from $A_{\mathrm{rms}}$ (e.g., for LWP 1, $A_{M}=0.53$ instead of 1.0). In summary, it is not easy to link the distribution of the modulation amplitudes to a particular type of MCVs owing to the lack of systematic survey. However, even in Figure 8 with selected samples of IPs and polars, the period distribution of the 10 periodic sources strongly favors polars.

Unlike the period distribution, the relatively hard X-ray spectra of the periodic sources in the LW imply that these systems are likely IPs. The quantile diagrams in Figure 9 illustrate the overall spectral hardness of the periodic sources (red) in comparison with the rest of the X-ray sources (black dots, net counts $\geqslant 50$ ) in the LW. Figures 9(a) and 9(b) overlay a set of simple power law and APEC model grids, respectively, over the same data points. The eight periodic sources found in the Sgr A* field are also shown in (green) crosses (M03b). All of the periodic sources show an intrinsically hard X-ray spectrum, similarly to the periodic sources in the Sgr A* field.

IPs tend to show harder X-ray spectra, which are associated with higher accretion rates and weaker magnetic fields (Ramsay $\&$ Cropper 2004). For instance, in the case of polars, the

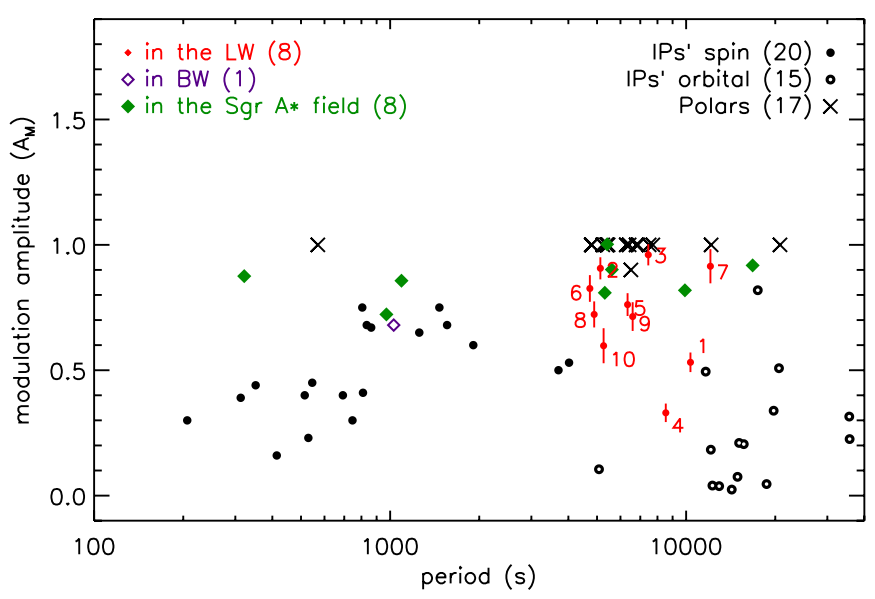

Figure 8. X-ray modulation $\left(A_{M}\right)$ vs. the period distribution of literatureselected IPs (black closed circles for spin periods and black open circles for orbital periods), polars (black " $\times$ "s), and the periodic sources in the LW (red circles), BW (purple diamond), and the Sgr A* field (green diamonds). References: orbital periods of IPs: Parker et al. (2005). Spin periods of IPs: GK Per, HT Cam, EX Hya, RX J1548.2, AO Psc, V1223 Sgr, AE Aqr-Evans \& Hellier (2005), V405 Aur-Evans \& Hellier (2004), FO Aqu-Evans et al. (2004), PQ Gem-James et al. (2002), IGR J15094-Butters et al. (2009), UU Col-de Martino et al. (2006), WX Pyx-Schlegel (2005), XY ARI-Salinas \& Schlegel (2004), T Leo-Vrielmann et al. (2004), TV Col-Rana et al. (2004), V1062 Tau-Hellier et al. (2002a), 1WGA J1958.2-Norton et al. (2002), YY Dra-Szkody et al. (2002), V709 Cas-Norton et al. (1999). Polars: V1309 Ori-Schwarz et al. (2005), EK UMa-Beuermann et al. (2009), HU Aqr-Schwarz et al. (2009), V2301 Oph-Ramsay \& Cropper (2007), SDSS J015543-Schmidt (2005), EP Dra-Ramsay et al. (2004a), OY Car-Wheatley \& West (2003), DP Leo-Ramsay et al. (2001), RX J1846.9-Schwarz et al. (2002), V407 Vul-Marsh \& Steeghs (2002), CE Gru-Ramsay \& Cropper (2002), V1432-Rana et al. (2005), V347 Pav, GG Leo, EU UMa-Ramsay et al. (2004b), and RX J1002-19-Ramsay \& Cropper (2003).

(A color version of this figure is available in the online journal.)

$\mathrm{X}$-ray spectra are well described by a blackbody component with $k T<60 \mathrm{eV}$ and a two-temperature thermal plasma component with $k T_{1}=0.7-0.9 \mathrm{keV}$ and $k T_{2}=3-5 \mathrm{keV}$ (e.g., Ramsay et al. 2004c), whereas the X-ray spectra of IPs show a blackbody component with $k T>60 \mathrm{eV}$ and a one- or two-temperature thermal plasma component with $k T \geqslant 10 \mathrm{keV}$ (e.g., Anzolin 2008). Owing to the interstellar absorption in the LW field $\left(N_{\mathrm{H} 22}\right.$ $\sim 0.7$ ), the blackbody component is usually undetectable, but the spectral distinction of the plasma components between polars and IPs remains detectable in the Chandra X-ray band: in a quantile diagram, polars would lie in the upper left section of the $k T \sim 4-10 \mathrm{keV}$ line and IPs in the lower right section (Figure 9). Although the above description of the X-ray spectra of IPs and polars is oversimplified and without a systematic survey (e.g., LWP 6 is likely a polar, see Section 5.6), it is generally accepted that IPs exhibit a harder spectrum. For instance, a recent survey of hard X-ray sources ( $\gtrsim 15 \mathrm{keV})$ conducted by Swift/BAT and INTEGRAL/IBIS shows that the composition of MCVs in the hard X-ray band is predominantly IPs (Scaringi 2010).

The period range and the relative hard X-ray spectra of the LW periodic sources also resemble those of some of the periodic sources found in the $\mathrm{Sgr} \mathrm{A}^{*}$ field (green data points in Figure 9 and blue points in Figure 6 (a); M03b). Of eight periodic sources in the Sgr A* field, four are in the same period range as the LW periodic sources with a large modulation amplitude $\left(A_{\mathrm{RMS}}>\right.$ $40 \%$ or $A_{M}>72 \%$ ), and all of them show relatively hard $\mathrm{X}$-ray spectra. One of them was identified as a foreground polar, based on the light curve, and it lies at the line of $k T=10 \mathrm{keV}$; the rest lie in the $k T>10 \mathrm{keV}$ section. In the case of the 

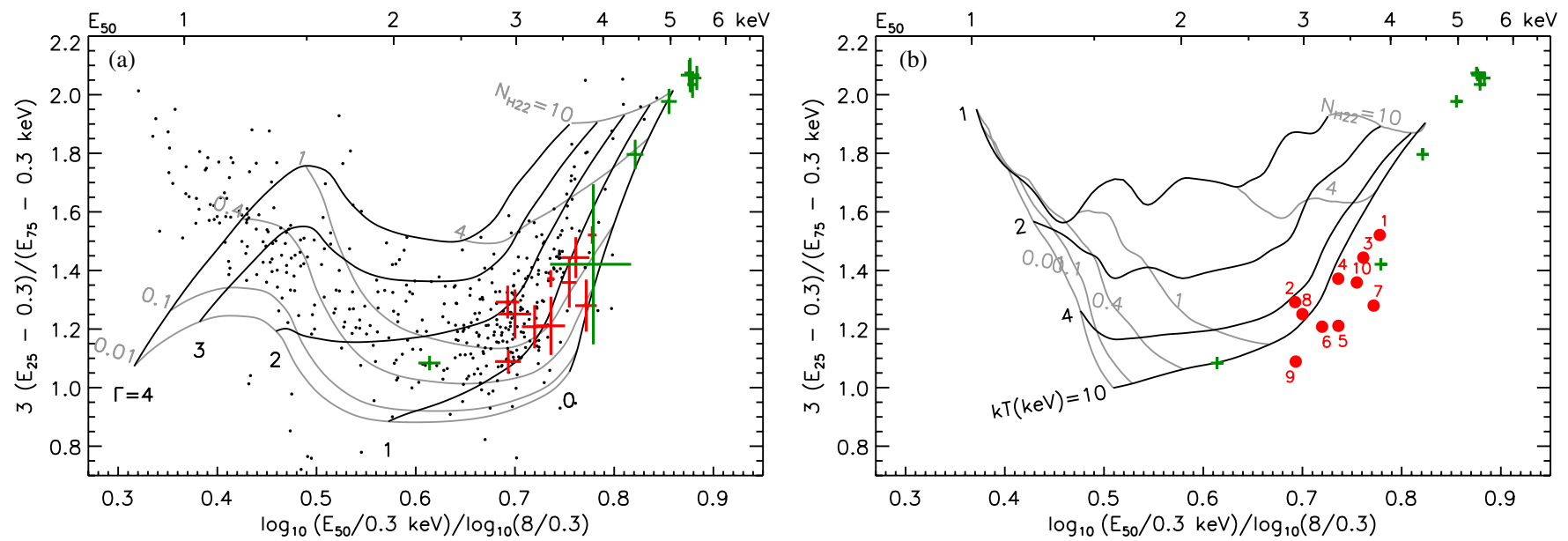

Figure 9. Quantile diagrams (H04; H09b) of the periodic X-ray sources in the LW (red) and the Sgr A* field (green). The same data points are plotted over a set of (a) power model grids or (b) APEC model grids. The (black) dots in (a) are the discrete X-ray sources in the LW with net counts greater than 50 in the $0.3-8$ keV band. The model grids are based on the response function at the aim point, which can be partially responsible for a small discrepancy between the spectral fit and quantile analysis in the parameter estimates for some sources (e.g., in LWP 9, $N_{\mathrm{H} 22}=0.2(3)$ from the spectral fit vs. 0.7(2) from the quantile analysis).

(A color version of this figure is available in the online journal.)

bulge X-ray sources in the Sgr A* field, the heavy interstellar absorption $\left(\sim 6 \times 10^{22} \mathrm{~cm}^{-2}\right)$ may complicate identification of the intrinsic X-ray spectra, but for the periodic sources in the LW $\left(\sim 7 \times 10^{21} \mathrm{~cm}^{-2}\right)$, there is little doubt that most of them exhibit intrinsically hard X-ray spectra.

An obvious question, then, is, what is the nature of the periodic X-ray sources found in the LW and the GCR, whose period distribution resembles polars' but X-ray spectra resemble IPs'?

First, one can consider that these are a rare type of polars with unusually hard X-ray spectra. Although uncertain, it is speculated that the origin of harder X-ray spectra in IPs relative to polars is related to the weaker magnetic field strength and the subsequently deeper penetration of the accretion stream into the WD surface. For instance, Cummming (2002) suggested that the relatively high accretion rate in IPs effectively buries the WD magnetic field, making them appear less magnetic, which in turn helps maintain the high accretion rate (e.g., V407 Vul; see Marsh \& Steeghs 2002). This idea nicely ties the dichotomy of the magnetic field strength between IPs and polars. Under this picture, one can imagine some unusual evolutionary scenarios from IPs to polars, where polars survive with a high accretion rate that can bury the magnetic fields, allowing a harder X-ray spectrum. If most of these periodic sources are located near the Galactic center $(8 \mathrm{kpc})$ as expected from the high absorption in their X-ray spectra and the high stellar density of the bulge, the $\mathrm{X}$-ray luminosities of these sources are estimated at the high end of the MCV range ( $\gtrsim 10^{32} \mathrm{erg} \mathrm{s}^{-1}$, see Table 3), which is also consistent with the above picture.

Second, a rare subclass of MCVs, nearly synchronous MCVs (ns-MCVs), perhaps also meet both of the observed properties - the period distribution and the relative hard X-ray spectra of the periodic sources in the LW. One can divide ns-MCVs into two subgroups - nearly synchronous IPs (ns-IPs) and asynchronous polars (APs). Both subgroups may exhibit similar $\mathrm{X}$-ray properties, but they probably represent different stages in the evolutionary path of MCVs.

First, APs, consisting originally of just four systems, which recently extended to eight according to the latest RK catalog (ver. 7.15), are traditionally considered as polars that are temporarily out of synchronization owing to a recent nova activity, which has altered their magnetic locking, giving $P_{s} /$ $P_{o} \sim 0.98-1.02$. Interestingly, it is speculated that APs exhibit a harder spectrum than normal polars, but with similar periods, as marked with purple diamonds in Figure 6(a). For instance, two of seven APs as opposed to two of 92 normal polars are found in the hard X-ray survey using INTEGRAL/IBIS, Swift/BAT, and Suzaku/HXD (Scaringi 2010).

Second, there are increasingly more IPs found near synchronization $\left(P_{s} / P_{o}>0.3\right)$. Starting with EX Hya, the list increases to six according to the RK catalog. Their orbital periods are predominantly around $1.5 \mathrm{hr}$ except for V697 Sco with a $4.5 \mathrm{hr}$ orbital period. According to the evolutionary model of Norton et al. (2008, hereafter N08), IPs start out with $P_{s} / P_{o}<0.1$, and as the systems evolve through magnetic lock, the orbital periods decrease and the spin periods increase, i.e., $P_{s} / P_{o}$ approaches 1 . Therefore, the orbital periods of ns-IPs will be clustered around or below the period gap near the end of the evolution, resembling the period distribution of polars more closely than that of usual, unsynchronized IPs.

As with polars exhibiting unusually hard X-ray spectra, the presence of ns-MCVs is very intriguing in terms of the evolutionary models of MCVs, challenging the conventional view of IPs with $P_{s} / P_{o} \lesssim 0.1$ and polars $\sim 0.98-1.02$. For instance, Paloma or RX J0524+42 (Pineault et al. 1987), recently identified as an AP, shows $P_{s} / P_{o} \sim 0.93$, and its relatively large de-synchronization $(\sim 7 \%)$ compared with conventional APs $(<2 \%)$ suggests that this system might represent the missing link of the evolutionary path between IPs and polars (Schwarz et al. 2007), rather than polars being out of synchronization temporarily owing to recent nova activities. As shown in Figure 6(a), the gap $(\sim 0.3-0.95)$ in the period ratio between IPs and polars is now more or less bridged by the recent discoveries of many ns-MCVs. It is speculated that some of these ns-MCVs transit between IPs and polars (e.g., V1025 Cen; see Hellier et al. 2002b).

According to $\mathrm{N} 08$, if the period ratio exceeds 0.6 , the only stable equilibrium is at synchronization $\left(P_{s} / P_{o}=1\right)$. Schwarz et al. (2007) suggest that the probability of finding ns-MCVs is very low, considering relatively short timescale for synchronization $(<1 \mathrm{Myr})$ compared with the lifetime of a CV ( 100 Myr; see also N08). Therefore, if many of the 
periodic sources in the LW are indeed ns-MCVs, it imposes another constraint on the evolutionary model or suggests an unusual environment of the Galactic bulge capable of harboring many such rare systems. A similar statement can be made for polars with unusually hard X-ray spectra.

The relative composition of source types in MCVs is highly biased, depending on search wavelengths. In the RK catalog, where most of the discoveries are based on optical/UV or longer wavelength bands, the relative ratio of IPs versus polars is close to 1 (e.g., 83 IPs versus 109 polars in Figure 6), whereas a hard X-ray survey ( $\gtrsim 15 \mathrm{keV}$ ) in Scaringi (2010) revealed 37 IPs and only 2 polars (both are APs). Therefore, if many of the periodic sources in the LW are indeed unusual polars or nsMCVs, it indicates that the Chandra X-ray band is well tuned for discovery of these rare MCVs.

Finally, we note that some of the observed X-ray properties of the periodic X-ray sources in the $\mathrm{LW}$ are shared by a group of quiescent low-mass X-ray binaries (qLMXBs). For instance, Swift J1353.5-0127, recently observed in an outburst as a black hole $(\mathrm{BH})$ transient, may have a relatively short orbital period ( $\sim 2 \mathrm{hr}$; see Casares et al. 2011) and exhibit a hard X-ray spectrum (Krimm et al. 2011). The observed overabundance of X-ray transients within 1 pc of Sgr A* (e.g., four out of seven within 20 pc; see Muno et al. 2005) implies that a large number of qLMXBs may be present as dark stellar remnants within 1 pc of Sgr A* (Schodel et al. 2007). However, CVs (and MCVs) are expected to be dominantly more abundant than these $\mathrm{BH}$ transients, and the periodic X-ray sources in the LW do not exhibit any strong outbursts in the $1 \mathrm{Ms}$ exposure spanned over 3 years (e.g., out of seven X-ray transients in the Sgr A* field, three or four sources have been observed in outbursts in each year; see Degenaar \& Wijnands 2009, 2010); it is reasonable to think that the large fraction of the periodic sources in the LW are in fact MCVs.

\subsection{Periodic Source Content in the GCR $X$-Ray Source Population}

In this section, we estimate the total number of periodic $\mathrm{X}$-ray sources in the LW through completeness simulations for periodicity detection. We have generated 500 synthetic light curves for a given set of X-ray modulation parameters including modulation period and amplitude, net count, and background count. Then we run the same detection algorithm to see how often the synthetic light curves are detected as periodic. This calculates $P_{\text {det }}$ for the given set of parameters. Here "detection" is simply defined as $P_{\text {FAP }}$ (Fix) $<1 \%$. ${ }^{11}$ The simulation is designed to match the data set observed in Epoch 4 with the same GTI gaps, and the simulated time tags are generated according to the barycenter-corrected CCD readout time cycles. The initial set of simulations indicates that the detection results do not depend on the given period range (100 s to $10 \mathrm{hr}$ ) (Figure 7 in Section 6.1), so we fix the period at $5432.1 \mathrm{~s}$ and varied the rest of the parameters. The simulated modulation amplitude $\left(A_{0}\right)$ ranges from $10 \%$ to $100 \%$ in $10 \%$ increments. The simulated net counts of sources are 50,100,150, 200,500,1000, and 3000, and the background counts are 30, 50, 100, 200, 500, and 1000 .

Using these simulation results, which cover most of the parameter space for the sources found in the LW, we estimate the periodicity detection probability $\left(P_{\mathrm{det}}\right)$ of the 381 sources with net counts $\geqslant 100$ in Epoch 4 . Since we do not know a priori the

\footnotetext{
11 The result in this section is consistent with that acquired with "detection" defined as $P_{\text {FAP }}($ Var $)<1 \%$.
}

distribution of modulation amplitude and the observed periodic sources show a wide range of the modulation amplitude $\left(A_{0}\right)$, we randomly assign a modulation amplitude $\left(A_{0}\right)$ to each source, assuming a uniform distribution from 0.1 to 1 . We repeat the interpolations 10,000 times (i.e., 10,000 sets of simulations), each with random assignments of modulation amplitude to cover the full modulation range for every source.

Figure 10 shows an example result, using 203 sources with net counts $\geqslant 100$ and $E_{50} \geqslant 2.5 \mathrm{keV}$ (hard X-ray sources, see also case 3 in Table 5) under the assumption that these sources are all periodic with a uniform distribution of the modulation amplitude. Figure 10(a) shows the expected number of sources with detectable periodicity in the two-dimensional phase space of net counts versus modulation depth for hard X-ray sources. The absolute number in each cell of the phase space depends on the cell size, so what matters here is a relative variation from cell to cell and the total number. The result shows that we expect to detect periodicity of 21(3) sources if all of the 203 sources are periodic with a uniform distribution of modulation amplitude.

Figures 10(b) and 10(c) show the same result (differential and accumulated) as a function of net count. The source distribution with detectable periodicity (red) indicates that the detection probability for sources with net counts less than 150 is very low. ${ }^{12}$ Since we only detected nine periodic sources with $P_{\text {FAP }}$ $($ Fix $) \leqslant 1 \%$, this result indicates that there should be about $87_{-12}^{+17}$ periodic sources, which is $43_{-7}^{+9} \%$ of the 203 sources. However, since the detection probability drops significantly at net counts below 200, we cannot make a reliable assessment of the faint periodic source population. If we limit our detection probability at $1 \%$, the dotted line in Figure 10(b) shows the effective population of the sources we can explore. Accordingly, the actual estimates of the periodic population can be as low as the dotted green line in Figure 10(c). Therefore, in the worstcase scenario where most of the sources with net counts below 200 are not periodic, the estimated periodic source population is $50_{-7}^{+10}$, which is about $24_{-4}^{+5} \%$ of the hard X-ray sources.

Figure 10(d) shows the accumulated percentage of the periodic sources in the LW as a function of net count. Figure 10(d) shows a gradual increase of the fractional periodic sources as net counts decrease to 200 from 500. This observed variation of the fractional periodic sources with net counts is consistent with the fact that the X-ray fluxes of the majority of MCVs are below $10^{32-33} \mathrm{erg} \mathrm{s}^{-1}$ (Heinke et al. 2008) since the observed periodic sources are at the high end of the X-ray luminosity distribution if they are in the bulge. It also implies that the true percentage of the periodic sources with net counts less than 200 is likely higher than the value (43\%) at net count $\sim 200$.

Table 5 summarizes the simulation results that estimate the total number of periodic sources including unidentified ones among the sources found in the LW. We repeated the analysis for four sub-sets of the sources, which cover samples only with nonCCD-edge sources and hard X-ray sources $\left(E_{50} \geqslant 2.5 \mathrm{keV}\right)$. In this table, we show the results for sources with net counts greater than 200, where we have a sufficiently high detection probability of periodicity given the number of sources. The case using non-CCD-edge sources enables an estimate of the systematic error in our selection procedure of periodic sources since the observed periodicity of the sources that fall near a CCD edge can be falsely discredited. The case using only hard X-ray sources should be a better representative of the bulge sources since they exclude the majority of the foreground soft sources.

\footnotetext{
12 This is an oversimplification since $P_{\text {det }}$ also depends on the background
} counts. See LWP 7 in Table 2 and Section 5.7. 

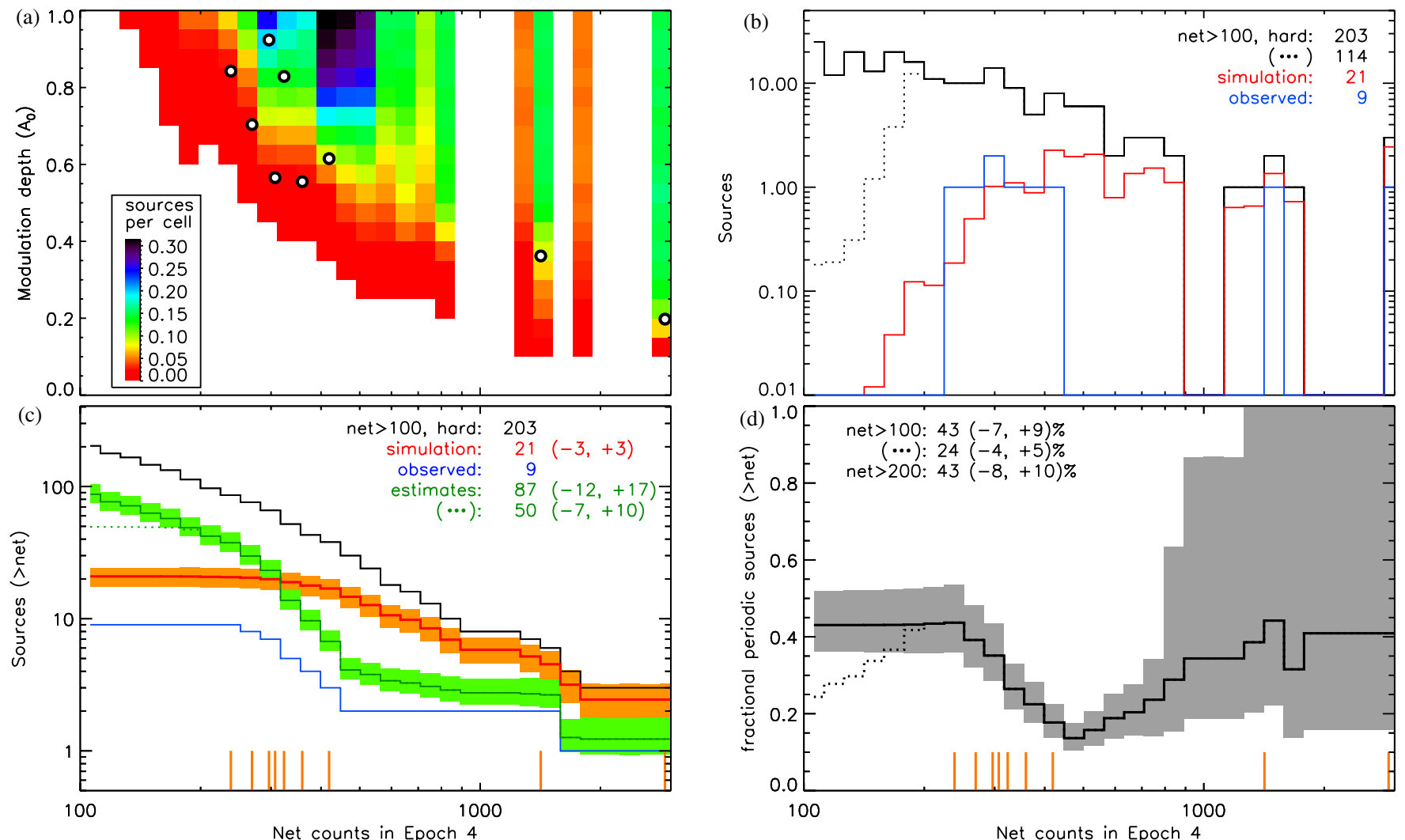

Figure 10. Completeness study for periodicity detection for hard X-ray sources ( $E_{50} \geqslant 2.5 \mathrm{keV}$; see also case 3 in Table 5). (a) The (relative) expected distribution of detectable periodic sources based on the completeness simulation under the assumption of all the sources being periodic with the uniform distribution of modulation amplitudes. The open circles show the 9 periodic sources with net counts $\geqslant 200$ in the LW. (b) The number of the sources with detectable periodicity (red) as a function of net count if all the sources (black) are periodic in comparison with the observed periodic sources (blue). The dotted line indicates the testable sample of the population if we limit the detection sensitivity at $1 \%$. (c) The accumulated number of the detectable sources (red) if all the sources are periodic (black). Given the observed periodic sources (blue), we also show the expected total population of periodic sources (green). Below 200 counts, the detection sensitivity is too low to make a reliable assessment of the hidden population of periodic sources (dotted green lines). The shade for green and blue indicates the statistical errors of the simulation results and estimates. (d) The fractional percentage (solid line) of the periodic sources, which is the ratio of the green to black lines in (c). The dotted line shows a conservative limit below 200 counts owing to lack of detection sensitivity, but the rapid rise of the percentage of periodic sources as the count approaches from 500 to 200 indicates that the trend will continue at low counts, where the majority of the bulge MCV populations lie (see Section 6.2).

(A color version of this figure is available in the online journal.)

Table 5

Estimation of the Total Number of Periodic Sources in the LW by Completeness Simulations

\begin{tabular}{|c|c|c|c|c|c|c|c|c|}
\hline \multirow{3}{*}{$\begin{array}{l}(1) \\
\text { Cases }\end{array}$} & \multirow{3}{*}{$\begin{array}{c}(2) \\
\text { Source Selection } \\
(\text { net } \geqslant 200 \text { in Epoch } 4)\end{array}$} & \multirow{3}{*}{$\begin{array}{c}\text { (3) } \\
\text { Total } \\
\text { Sources }\end{array}$} & \multirow{2}{*}{\multicolumn{4}{|c|}{ Periodic X-Ray Sources }} & \multirow{2}{*}{\multicolumn{2}{|c|}{$\begin{array}{c}(8) \\
\text { Alternative Estimates } \\
\end{array}$}} \\
\hline & & & & & & & & \\
\hline & & & Observed & Detectable & Estimated Total & Percentage & Estimated Total & Percentage \\
\hline & All & 1530 & 9 & $28(-4$, & $48(-6$ & $32(-5$ & 48( & 32 \\
\hline & Non-edge & 113( & 0 & $21(-4,+4)$ & $43(-$ & $38(-7$ & & $38(12)$ \\
\hline & $E_{50} \geqslant 2.5 \mathrm{keV}$ & $96(10)$ & 9 & $21(-3,+3)$ & $42(-6$ & $43(-8$, & $42(12)$ & $43(13)$ \\
\hline & $E_{50} \geqslant 2.5 \mathrm{keV}$, non-edge & $69(8)$ & 8 & $16(-3,+3)$ & $35(-6,+8)$ & $51(-11,+14)$ & $35(11)$ & $51(16)$ \\
\hline
\end{tabular}

Notes. Columns: (1) Case number. (2) Source selection criteria. We limit the sample to sources with $\geqslant 100$ net counts in Epoch 4. (3) The total number of sources that meet the source selection criteria. (4) The number of the observed periodic sources from Table 2. (5) The estimated number of the detectable periodic sources by simulations under the assumption that all the sources are periodic with a uniform distribution of modulation amplitude. (6) The estimated total number of the periodic sources in source selection: (3) $\times(4) /(5)$. (7) The percentage of periodic sources in the selection: (6)/(3). (8) An alternative (error) estimate of the total number of periodic sources and (9) their percentage using negative binomial distribution, where, for the observed periodic sources, $N$, and the non-detection probability, $p$, given by the simulations (in case $3, N=9,1-p=21 / 100$ ), the estimated unidentified periodic sources and its variance are $N p /(1-p)$ and $N p /(1-p)^{2}$, respectively.

A simple ratio argument between the observed periodic sources and simulation results (Columns 6 and 7 in Table 5) indicates that about $32 \%-38 \%$ of the sources in the LW should be, in fact, periodic and the percentage increases to $43 \%-51 \%$ for the hard X-ray sources. The errors of these estimates are cal- culated from the variances of the total sample sizes (Column 3) and the ranges of the detectable periodic sources (Column 5), provided that at least the observed number of periodic sources (Column 4) is present in each case. Alternatively, one can estimate the errors for the total number of the periodic sources 
according to negative binomial distribution (Columns 8 and 9 in Table 5), which provides the variance estimates of the unidentified (and thus total) number of periodic sources.

The result indicates that a large fraction of the bulge X-ray sources ( $\gtrsim 30 \%-40 \%$ of hard X-ray sources even with errors) found in the GCR should be periodic. This provides a first direct evidence for the presence of a large population of MCVs in the bulge X-ray sources, which supports that MCVs constitute a large majority of the bulge X-ray sources.

\section{SUMMARY}

We have found 10 periodic X-ray sources in the LW from the 1 Ms exposure of the Chandra ACIS-I instrument. The overall period distribution, X-ray luminosity, and spectral properties of these periodic sources and eight periodic sources found in the Sgr A* field (M03b) fit the general description of MCVs, supporting the argument that MCVs are the major constituent of the bulge X-ray sources. However, when inspecting the details of these X-ray properties-period distribution that resembles the polars' and the hard X-ray spectra that resemble the IPs'-we discover that these periodic sources may fit into rare MCVs, polars with unusually hard X-ray spectra or ns-MCVs. The completeness tests indicate that at least about $40 \%$ of the hard $\mathrm{X}$-ray sources in the LW are periodic, which is a first direct evidence of a large MCV population in the bulge X-ray sources.

Given the difficulty of identifying the nature of the bulge X-ray sources in the GCR due to high stellar density, large distance, and heavy obscuration, we recommend continuous $\mathrm{X}$-ray monitoring of the GCR for future discoveries of periodicity and unusual variability. Considering the importance of these unusual MCVs in understanding the evolutionary path of MCVs and their potential connection to thousands of the bulge $\mathrm{X}$-ray sources, we also encourage systematic in-depth X-ray studies of a similar kind (e.g., there are only 13 ns-MCVs with $P_{s} / P_{o}>3$ in the RK catalog) to establish a demographic profile of their X-ray properties if any.

We thank Dr. Silas Laycock for the Magellan/IMACS observation and the initial data processing of the LW. We also thank Dr. Brandon Kelly for suggesting negative binomial distribution. This work is supported in part by NASA/Chandra grants GO6-7088X, GO7-8090X, and GO8-9093X. We also thank Chandra X-ray Center (CXC) for their support in publication. Finally, we thank the anonymous referee for useful comments.

\section{REFERENCES}

Anzolin, G. 2008, A\&A, 489, 1243

Beuermann, K., Diese, J., Paik, S., et al. 2009, A\&A, 507, 385

Bretthorst, G. L. 1988, Bayesian Spectrum Analysis and Parameter Estimation (Berlin: Springer)

Butters, C. H., Norton, A. J., Mukai, K., \& Barlow, E. J. 2009, A\&A, 498, L17

Casares, J., Torres, M. A. P., Negueruela, I., et al. 2011, ATel, 3206

Cumming, A. 2002, MNRAS, 333, 589

Degenaar, N., \& Wijnands, R. 2009, A\&A, 495, 547

Degenaar, N., \& Wijnands, R. 2010, A\&A, 524, 69

de Martino, D., Matt, G., Mukai, K., et al. 2006, A\&A, 454, 287

Dolphin, A. E. 2000, PASP, 112, 1383

Evans, P. A., \& Hellier, C. 2004, MNRAS, 353, 447
Evans, P. A., \& Hellier, C. 2005, in ASP Conf. Ser., 330, The Astrophysics of Cataclysmic Variables and Related Objects, ed. J.-M. Hameury \& J.-P. Lasota (San Francisco, CA: ASP), 165

Evans, P. A., \& Hellier, C. 2007, ApJ, 663, 1277

Evans, P. A., Hellier, C., Ramsay, G., \& Cropper, M. 2004, MNRAS, 349, 715

Freeman, P. E., Kashyap, V., Rosner, R., \& Lamb, D. Q. 2002, ApJS, 138, 185

Grindlay, J. E., Hong, J., Zhao, P., et al. 2005, ApJ, 635, 920

Heinke, C. O., Ruiter, A. J., Muno, M. P., \& Belczynski, K. 2008, in AIP Conf. Proc. 1010, A Population Explosion: The Nature \& Evolution of X-ray Binaries in Diverse Environments, ed. R. M. Bandyopadhyay, S. Wachter, D. Gelino, \& C. R. Gelino (Melville, NY: AIP), 136

Hellier, C., Beardmore, A. P., \& Mukai, K. 2002a, A\&A, 389, 904

Hellier, C., Wynn, G. A., \& Buckley, D. A. H. 2002b, MNRAS, 333, 84

Hong, J., Schlegel, E. M., \& Grindlay, J. E. 2004, ApJ, 614, 508 (H04)

Hong, J., van den Berg, M., Grindlay, J. E., \& Laycock, S. 2009a, ApJ, 706, 223 (H09a)

Hong, J., van den Berg, M., Laycock, S., Grindlay, J. E., \& Zhao, P. 2009b, ApJ, $699,1053(\mathrm{H} 09 \mathrm{~b})$

Hong, J., van den Berg, M., Schlegel, E. M., et al. 2005, ApJ, 635, 907 (H05)

James, C. H., Ramsay, G., Cropper, M., et al. 2002, MNRAS, 336, 550

Koenig, X., Grindlay, J. E., van den Berg, M., et al. 2008, ApJ, 685, 463

Krimm, H., Kennea, J. A., \& Holland, S. T. 2011, ATel, 3142

Laycock, S., Grindlay, J., van den Berg, M., et al. 2005, ApJ, 634, L53 (L05)

Leahy, D. A., Darbro, W., Elsner, R. F., et al. 1983, ApJ, 266, 160

Marsh, T. R., \& Steeghs, D. 2002, MNRAS, 331, L7

Muno, M. P., Baganoff, F. K., Bautz, M. W., et al. 2003a, ApJ, 589, 225 (M03a)

Muno, M. P., Baganoff, F. K., Bautz, M. W., et al. 2003b, ApJ, 599, 465 (M03b)

Muno, M. P., Bauer, F. E., Baganoff, F. K., et al. 2009, ApJS, 181, 110 (M09)

Muno, M. P., Pfahl, E., Baganoff, F. K., et al. 2005, ApJ, 622, L113

Norton, A. J., Beardmore, A. P., Allan, A., \& Hellier, C. 1999, A\&A, 347, 203

Norton, A. J., Butters, O. W., Parker, T. L., \& Wynn, G. A. 2008, ApJ, 672, 524

Norton, A. J., Quaintrell, H., Katajainen, S., et al. 2002, A\&A, 384, 195

Parker, T. J., Norton, A. J., \& Mukai, K. 2005, A\&A, 439, 213

Pineault, S., Landecker, T. L., \& Routledge, D. 1987, ApJ, 315, 580

Protassov, R., van Dyk, David A., Connors, A., et al. 2002, ApJ, 571, 545

Ramsay, G., \& Cropper, M. 2002, MNRAS, 335, 918

Ramsay, G., \& Cropper, M. 2003, MNRAS, 338, 219

Ramsay, G., \& Cropper, M. 2004, MNRAS, 347, 497

Ramsay, G., \& Cropper, M. 2007, MNRAS, 379, 1209

Ramsay, G., Bridge, C. M., Cropper, M., et al. 2004a, MNRAS, 354, 773

Ramsay, G., Cropper, M., Crdova, F., et al. 2001, MNRAS, 326, L27

Ramsay, G., Cropper, M., Mason, K. O., et al. 2004b, MNRAS, 347, 95

Ramsay, G., Cropper, M., Wu, K., et al. 2004c, MNRAS, 350, 1373

Rana, V. R., Singh, K. P., Barrett, P. E., \& Buckley, D. A. H. 2005, ApJ, 625, 351

Rana, V. R., Singh, K. P., Schlegel, E. M., \& Barrett, P. 2004, AJ, 127, 489

Revnivtsev, M., Sazonov, S., Churazov, E., et al. 2009, Nature, 458, 1142

Revnivtsev, M., Sazonov, S., Forman, W., Churazov, E., \& Sunyaev, R. 2011, MNRAS, 414, 495

Revnivtsev, M., van den Berg, M., Burenin, R., et al. 2010, A\&A, 515, A49

Ritter, H., \& Kolb, U. 2003, ApJ, 404, 301

Salinas, A., \& Schlegel, E. M. 2004, AJ, 128, 1331

Scargle, J. D. 1982, ApJ, 263, 835

Scargle, J. D. 1998, ApJ, 504, 405

Scaringi, S., Bird, A. J., Norton, A. J., et al. 2010, MNRAS, 401, 2207

Schlegel, E. M. 2005, A\&A, 433, 635

Schmidt, G. D. 2005, ApJ, 620, 422

Schodel, R., Eckart, A., Alexander, T., et al. 2007, A\&A, 469, 125

Schwarz, A. D., Schwope, A. D., Staude, A., et al. 2007, A\&A, 473, 511

Schwarz, R., Greiner, J., Tovmassian, G. H., Zharikov, S. V., \& Wenzel, W. 2002, A\&A, 392, 505

Schwarz, R., Reinsch, K., Beuermann, K., \& Burwitz, V. 2005, A\&A, 442, 271

Schwarz, R., Schwope, A. D., Vogel, J., et al. 2009, A\&A, 496, 833

Szkody, P., Nishikida, K., Erb, D., et al. 2002, AJ, 123, 413

van den Berg, M., Grindlay, J., Laycock, S., et al. 2006, ApJ, 135, L647

van den Berg, M., Hong, J. S., Grindlay, J. E., et al. 2009, ApJ, 700, 1702 (B09)

Vrielmann, S., Ness, J.-U., \& Schmitt, J. H. M. M. 2004, A\&A, 419, 673

Warner, B. 1995, Cataclysmic Variable Stars (Cambridge: Cambridge Univ. Press)

Wheatley, P. J., \& West, R. G. 2003, MNRAS, 345, 1009

Zhao, P., Grindlay, J. E., Hong, J. S., et al. 2005, ApJS, 161, 429 